(Aus dem Physiologischen Institute der Deutschen Universität in Prag [Vorstand: Prof. Dr. A. Tschermak].)

\title{
Beiträge und kritische Studien zur Heterophoriefrage auf Grund systematischer Untersuchungen.
}

\author{
Von
}

Dr. Max Heinrich Fischer,

I. Assistenten am Institute.

Mit 12 Textabbildungen.

Im Anschlusse an unsere, von $A$. Tschermak angeregten, ausführlichen Studien über die absolute Lokalisation bei normalen Binokularsehenden $\left[M . H\right.$. Fischer $\left.\left.^{21}\right)\right]$ ergaben sich mancherlei Probleme, deren Bearbeitung mit exakten subjektiven Untersuchungsmethoden*) die vielerorts leider noch nicht genügend gewürdigt werden - nicht allein für den Physiologen und Theoretiker, sondern auch für den praktischen Ophthalmologen von nicht geringer Bedeutung ist, zumal daraus vielfach neuere wohlbegründete Auffassungen erwachsen. So erschien es unter anderem von Interesse, Untersuchungen in dreierlei Richtung an einem größeren Materiale vorzunehmen: über die Primärstellung, die Ruhestellung und die Abblendungsstellung der Augen. Wir wählten zunächst aus noch später zu diskutierenden Gründen systematische Untersuchungen über die letztere bei einer größeren Zahl von Individuen. Wenn hier auch nicht beabsichtigt ist, eine erschöpfende Darstellung der schier unübersehbaren Literatur zu geben, so sei doch erlaubt, auf das Wichtigste hinzuweisen und eine strikte Formulierung mancher heute ganz sicher festgelegten Begriffe zu geben, die sich schon deswegen nicht erübrigt, weil noch vielfach in dieser Nomenklatur arger Mißbrauch getrieben wird.

\section{Die Primärstellung der Augen.}

Wie bekannt ist die Primärstellung seit langem genau kinematisch charakterisiert durch das Listing sche Gesetz, welches lautet: „daß es eine, aber auch nur eine ganz bestimmte Lage des Augapfels in der Orbita und also auch nur eine bestimmte Stellung der Gesichtslinie

*) Über die Bedeutung des exakten Subjektivismus in der Sinnesphysiologie siehe A. Tschermal ${ }^{5}$ ). 
relativ zu den drei Hauptebenen des Kopfes gibt, aus welcher heraus die Gesichtslinie in jeder beliebigen ebenen Bahn durch einfache Drehung und also ohne gleichzeitige Rollung bewegt werden kann"**). Das Listingsche Gesetz entspricht dem Prinzip der vermiedenen Rollung um die Gesichtslinie und damit der vermiedenen Scheinbewegung**). Würden nämlich die Augen eine Rollung um die Gesichtslinie erfahren, so müßte dabei unvermeidlich, wie bei jeder unwillkürlichen Bewegung ***), eine gegensätzliche Scheindrehung der Sehdinge auftreten. Betreffs neuerer Bearbeitungen des Listingschen Gesetzes und des Problems der Primärstellung sei hier nur auf die ausgezeichneten mathematischen. Darlegungen $O$. Fischers ${ }^{15}$ ) hingewiesen. Es sei nur noch einmal betont, daß also der Begriff "Primärstellung der Augen" als etwas ganz Bestimmtes heute vollständig außer Diskussion steht, Es muß daher als ganz unrichtig und nur verwirrend bezeichnet werden, wenn Reddingius $^{16}$ ) die Lage der Augen mit divergenten Gesichtslinien beim Blick in die Ferne - von ihm zugleich Ruhelage genannt - als Primärstellung bezeichnet und die kinematische "Primärstellung" als eine durch Konvergenz erreichte Sekundärstellung auffaßt. Eine sehr exakte Methode zur Aufsuchung der Primärstellung ist gegeben durch die Heringsche Nachbildmethode [E. Hering ${ }^{2}$ ), spez. S. 74-83], deren nähere Ausführung als nicht hierhergehörig übergangen sei. Nur sei betont, daß man zweckmäßigerweise (nach A. Tschermak) zur Nachbilderzeugung einen drehbaren radiären Streifen oder Kreuzesarm wählt, den man in den dann später mit dem Blicke zu durchmessenden Radianten des Gesichtsfeldes einstellt. Entspricht die Ausgangsstellung der „Primärstellung ${ }^{6}$, dann - aber auch nur dann - bewahrt das Nachbild gegenüber dem Radianten sozusagen Treue, während es sonst von ihm durch Schiefwerden abweicht. Nur nebenbei sei erwähnt, daß beim normalen Binokularsehenden die Augen ungefähr die „Primärstellung" einnehmen, wenn man in aufrechter Kopf- und Körperhaltung mit parallelen Gesichtslinien vor sich hin in die Ferne blickt, wie Verf. für sich durch wiederholte Prüfung nachweisen konnte. Diese allgemeinen Bemerkungen über das Listingsche Gesetz und die Primärstellung mögen hier genügen. Nicht unerwähnt darf aber bleiben, daß neuere langwierige Arbeiten, die in unserem Institute unter der Leitung A. Tschermaks über das Problem der Primärstellung im allgemeinen durchgeführt wurden und werden, mit Bestimmtheit erwiesen haben, daß die Kopfstellung für das Bewegungsgesetz der Augen nicht bedeutungslos ist,

*) E. Hering ${ }^{2}$, S. 470 ; ${ }^{2}$ ) S. 64.

**) E. Hering ${ }^{2}$ ), S. 106 .

***) Man denke beispielsweise an die deutlich auftretenden Scheinbewegungen bei Freigabe eines verdeckten Auges, wenn dasselbe durch die Fusion aus seiner Abblendungsstellung zur binokularen Einstellung gezwungen wird [A. Graefe $\left.\left.{ }^{17}\right)\right]$. 
șondern daß es auch eine ganz bestimmte als „Kopf-Primärstellung“ charakterisierbare Stellung des Kopfes gibt, für welche (ebenso wie für die davon rein lotrecht oder rein wagrecht verschiedenen Sekundärstellungen) das Listing sche Gesetz der rollungsfreien Augenbewegung gilt, während es für Bewegungen von jeder Tertiärstellung des Kopfes aus nicht zutrifft. Doch sei die weitere Behandlung dieses schwierigen Themas einer späteren Institutsarbeit vorbehalten. Diese neuerliche Komplikation läßt es neben der Umständlichkeit der Beobachtung an sich begreiflich erscheinen, daß Massenuntersuchungen über die Primärstellung der Augen und das Listingsche Gesetz absolut undurchführbar sind. In den wenigen genau untersuchten Fällen (speziell ich selbst, A. Tschermak, $G$. Schubert) hat sich unverkennbar eine deutliche Differenz von Primärstellung und ,Ruhelage" (bestimmt auf Grund der Ermittlung von S.GI.H. und S.G.V.) ergeben.

\section{Die „Ruhelage“" der Augen.}

Man hat unter Ruhelage der Augen in der physiologischen und ophthalmologischen Literatur bisher so vielerlei verstanden, daß eigentlich bis heute noch keine richtige Klarheit darüber besteht*). Ein kurzer historischer Rückblick sei hier geboten. A. Graefe ${ }^{10 a, 1^{10 b}}$ ) nennt absolute Ruhestellung der Augen jene Stellung, in welcher sich die Bulbi befinden, wenn bei aufrechter Kopfhaltung gerade nach vorne in die Ferne gesehen wird, wobei angenommen wird, daß sich sämtliche Augenmuskeln im Ruhezustande befinden. Es sei das dieselbe Stellung der Augen, in welcher sie sich gewöhnlich nach dem Tode oder plötzlicher vollkommener Paralyse - also bei Wegfall jedweder Innervation befinden. Hansen $\left(\right.$ rrut $^{19}$ ) versteht unter anatomischer Ruhelage $\left.{ }^{* *}\right)$ jene Stellung der Augen, welche sie knapp nach dem Tode noch vor Eintreten der Totenstarre oder nach vollständiger Lähmung aller Augenmuskeln im Leben einnehmen. Dabei wurde meistens eine Divergenzstellung der Gesichtslinien gefunden, seltener Parallelstellung, ganz selten Konvergenz. Der Winkel $\gamma$ konnte dabei natürlich nicht berücksichtigt werden, wenn die Untersuchungen an Leichen angestellt wurden. $A . v$. Graefe ${ }^{30}$ ) und $H$. Oppenheimer ${ }^{53}$ ) geben ähnliche Befunde bei totaler Ophthalmoplegie - untersucht durch Distanzmessungen der Doppelbilder - an. Bielschowsky ${ }^{54}$ ) bezeichnet als absolute Ruhelage die Lage des Einzelauges, wie sie bei Fehlen jeglicher nervöser Beeinflussung lediglich durch die topographisch-anatomischen Verhältnisse innerhalb der Orbita, also durch rein mechanische Faktoren bedingt ist; er bemerkt, daß während des Lebens eine exakte Bestimmung dieser anatomischen Ruhelage natürlich undurchführbar ist, weil ja die nervöse

*) Siehe die zusammenfassende Darstellung bei F. B. Hofmann ${ }^{20}$ ).

**) Untersuchungen über die Leichenstellung der Augen wurden bereits von $F_{i c k}^{50}$ ), Ruete ${ }^{51}$ ) und A. W. Volkmann ${ }^{52}$ ) durehgeführt. [Siehe Hering ${ }^{1}$ ), S. 517.] 
Beeinflussung der Augenmuskeln nicht vollständig auszuschalten ist. Die Frage der „relativen Ruhelage" der Augen wird später zu erörtern sein.

Es ist nun eine wichtige Frage, ob es zweckmäßig ist, wenn man überhaupt von einer Ruhelage der Augen sprechen will, dieselbe im Sinne vorgenannter Autoren aufzufassen. Das größte Interesse dürfte wohl die physiologische, funktionelle Ruhelage haben, wenn sich ein solcher Begriff formulieren läBt und die Möglichkeit gegeben ist, dieselbe zu. bestimmen. Die anatomische Ruhelage (Hansen Grut), mit der sich wohl die absolute Ruhelage im Sinne Bielschowskys so ziemlich deckt, dürfte innerhalb des normalen Lebens relativ selten, wenn überhaupt, vorkommen, man denke nur an eventuelle Divergenz. Gegen die absolute Ruhestellung $A$. Graefes ist einzuwenden, daß sich beim Blicke gerade. ans in die Ferne mit beiden Augen wohl meistens nicht sämtliche Augenmuskeln im Ruhezustande befinden können, sondern vielmehr einen ungleichmäßigen Grad von alktiver Spannung besitzen, da in der Mehrzahl der Fâlle die Abdeckung des einen Auges dazu führt, daß dieses die Finstellung "Gendeaus in die Ferne" verläßt und in eine davon bzw. von der Einstellung des anderen Auges versehiedene Lage übergeht. Die Einstellung "Geradeaus in die Ferne" ist also wenigstens für dieses Auge eine aktive Zwangslage, keine Ruhelage an Innervation. Fehlen einer solchen korrektiven Fusionsinnervation, d. h. Gleichbeit ron $A b$ blendungsstellung des Einzelauges und binokularer Einstellung, sog. Orthophorie, ist, wie bereits Bielschowsky ${ }^{54}$ ) (S. 72) ausgefüht hat, an normalen Binokularsehenden höchstens in $20-25 \%$ nachweisbar, eine Zahl, die durch unsere Unterswchungen eine noch größere Einsohränkung wird erfahren müssen. Es ist überhaupt fraglich, ob eine ideale Orthophorie jemals vorkommt; wenn ja, so wäre dies ein ganz seltener Ausnahmsfall. Inwieweit sich die Befunde bei totaler Ophthalmoplegie in unserem Sinne verwerten lassen, kann erst später dislkutiert werden.

Wir fassen die ,Ruhelage" der Augen mit A. Tschermak im physiologisch-funktionellen Binne als eine "tonische" Gleichgewichtslage auf, bei welcher sich sämtliche Augenmuskeln in einem Ninimaltonus (Minimalspannungszustande) befinden, und verstehen hier unter, "Tonus" in erster Linie den Endeffekt einer dauernden neurogenen Beeinflussung der Angenmuskeln, die natiurlich unserem Willen nicht unterstellt ist, wobei es zunächst gleichgültig ist, $o b$ in diesem Falle ein wabrer Tonus wie an der glatten Muskulatur vorliegt oder ein scheinbarer, $d$, h. ein schwacher dauernder Tetanus - bestehe er allein oder aufgesetat auf einen wahren Tonus*). Wahrscheinlich besteht neben dem neurogenen

*) Vgl. A. Tschermak, Bioelektrische Studien an der Magenmuskulatur; I. Mitteilung: Das Elektrogastrogramm (Egg) bei Spontanrhythmil des isolierten Frosehmagens. Pflügers Arch. f. d. ges. Physiol. 175, 105-186. 1919. Vgl. auch: Zur Lehre von der tonischen Innervation. Wiener klin. Wochensohr. 28, Nr.13. 1914. 
„Tonus" der Augenmuskeln noch eine myogene Komponente. Daß es überhaupt einen neurogenen "Tonus" der Augenmuskeln gibt, ist heute wohl nicht mehr zu bezweifeln. Schon E. Hering ${ }^{3}$ ) fand bei seinen Untersuchungen über die Muskelgeräusche des Auges bei ruhenden Augen am Bulbus ein Dauergeräusch, auf welches sich bei Willkürbewegungen Momentangeräusche superponieren, und war geneigt, dasselbe als den Ausdruck einer Dauerinnervation aufzufassen. Schließlich wies $P$. Hoff$m a n n^{55}$ ) am Kaninchen durch die elektrographische Methode mit dem Saitengalvanometer nach, daß von ruhenden Augenmuskeln - beim Kaninchen sind spontane Augenbewegungen überhaupt nur andeutungsweise vorhanden - ein Dauerstrom abzuleiten ist, welcher einen deutlich rhythmischen Charakter zeigt. Der Augenmuskeltonus sei demnach als ein schwacher Tetanus aufrufassen. Damit muß allerdings nach A. Tschermak die Möglichkeit offen gelassen werden, daß die an ,tonisierten" Augenmuskeln beobachteten Muskelgeräusche und bioelektrischen Rhythmen doch nur der Ausdruck einer zu einem eigentlichen Tonus hinzutretenden temporären Erregung seien. Sind doch auch an quergestreiften Muskeln Tonuszustände bekannt, welche nicht mit bioelektrischen Rhythmen oder ,Aktionsströmen" einhergehen. (Speziell $R . H . K a h n^{48}$ ) bezüglich des Umklammerungsreflexes an männlichen Fröschen.) Eine definitive Lösung der Tonusfrage können nur weitere Cntersuchungen an glatten und quergestreiften Muskeln, auch speziell an der Augenmuskulatur bringen!

$\mathrm{Da} B$ der echte oder sog. Tonus der Augenmuskeln durch Einwirkung vom Zentralnervensystem her erhalten wird, ist wohl sicher; ein sehr schwieriges Problem ist es allerdings, den Ursprungsort und die Natur dieser Einflußnahme näher bezeichnen zu wollen, es läßt sich diese Frage wohl heute nicht ganz lösen. Wir wären am ehesten geneigt, den sog. Tonus als Resultante von Dauereinwirkungen anzusehen, welche auf die Augenmuskeln in erster Linie von subcorticalen Zentren ausgeübt werden, wobei aber nicht vergessen werden darf, $\mathrm{daB}$ dieser sog. Tonus reflektorisch beeinflußbar ist durch Belichtung, durch Rezeptionen seitens des Labyrinthes und der Halsmuskulatur, wie insbesondere die interessanten und exakten neueren Untersuchungen von R. Magnus, A. de Kleijn und van der Hoeve 24, 36, 37, 38) gezeigt haben, und daß auch die Großhirn- wie die Kleinhirnrinde auf den sog. Tonus der Augenmuskeln modifizierend einwirken kann. Ophthalmoplegien gehen nach dieser Auffassung nur dann notwendig mit vollständigem Verlust des neurogenen Augenmuskeltonus einher, wenn sie nuclear oder peripher, also durch Erkrankungen distal von den Augenmuskelkernen, bedingt sind, während das bei supranuclearen nicht notwendig der Fall sein müßte. Dafür sprechen unter anderem die experimentellen Feststellungen von A. de Kleijn und R. Magnus ${ }^{21}$ ), welche bei ihren 
Untersuchungen über die Labyrinthreflexe auf die Augenmuskeln fanden, daßnoch, tonische "Beeinflußungen der Augenmuskeln von seiten derLabyrinthe zustande kommen, wenn dieVersuchstiere (in erster Linie Kaninchen und Katzen) sowohl des Großhirns als auch des Kleinhirns beraubt waren, daß also die Zentren für diese Reflexe im Hirnstamme liegen müssen*).

Es ist nun - wie sich später noch zeigen wird - sicher, daß die sog. tonische Gleichgewichtslage, die „Ruhelage" jedes Einzelauges nicht weitgehend von seiner ,anatomischen Ruhestellung" verschieden sein kann, aber ebenso sicher, daß sie mit dieser nicht ohne weiteres identifiziert werden darf, sondern als etwas prinzipiell Verschiedenes angesehen werden muß. Gewiß ist auch die ,Ruhelage "***) weitgehend abhängig von den topographisch anatomischen Verhältnissen in der Orbita, doch nicht allein; es kommt noch ein neuer Faktor hinzu, der sog. ,Tonus", der bestimmt an allen Augenmuskeln nicht in gleichmäßigem Grade bzw, in gleicher Wirksamkeit verteilt ist und dazu noch - wie früher vom Verfasser bereits gezeigt wurde - zeitlichen Schwankungen unterliegt. Man muß überhaupt, wie auch hier im Anschluß an $A$. Tschermak ausdrücklich betont sei, immer mit gewissen Unregelmäßigkeiten, Disharmonien oder Diskrepanzen und Asymmetrien in Bau und Leistung der Organismen als mit schion de norma gegebenen Faktoren rechnen; allerdings entziehen sich dieselben für gewöhnlich meist unserer Beobachtung und sind nur mit exakten Methoden bei spezieller Aufmerksamkeit nachweisbar. Wir haben für gewöhnlich - zum Teil dank besonderer korrektiver Momente - den Eindruck, als ob das Prinzip der Harmonie und Symmetrie gewahrt wäre. Rein geometrische Symmetrie in Form und Leistung ist nichtsdestoweniger als ganz besondere Seltenheit zu betrachten, wenn sie überhaupt je vorkommen sollte. - Der Hauptunterschied besteht also darin, daß die , Ruhelage im Gegensatse zur anatomischen Ruhestellung etwas Physiologisch-Funktionelles darstellt.

Wie kann man nun die ,Ruhelage" finden? Ganz exakt ist das wohl überhaupt nicht möglich. Doch wurde von uns eine Methode ausgearbeitet, die mit größtmöglichster Annäherung ziemlich gute Resultate liefert, allerdings immer nur für ein Einzelauge. Diese Methode, die bereits von $A . T_{s c h e r m a k}{ }^{6}$ ) ausführlich behandelt worden ist, ist allerdings an und für sich etwas umstänđlich und läßt sich wohl zu Massenuntersuchungen, wie sie der Ophthalmologe benötigt, kaum verwerten, sondern ist mehr oder weniger nur zur exakten Critersuchung einzelner Fälle anwendbar. Zur Klärung der Frage der "Ruhelage" der Augen

*) Hierher gehört auch der von Bielschowsky ${ }^{23}$ ), S. 164 erwäbnte Fall einer bilateralen seitlichen Blicklähmung duroh supranueleare Sebädigung, wo die Augen keine willkürliche Seitenwendung ausführen Konnten, aber durch Zustandsanderungen des Vestibula rapparates (Kopfdrehung usw.) dazu gebracht werden konnten.

**s) Unter" "Ruhelages (mit Anfuhrungszeichen) soll won nun ab immer die "tonisehe" Gleichgewichtstage verstanden werlen. 
und der absoluten Lokalisation sowohl beim normalen Binokularsehenden als auch beim Schielenden ist sie allerdings unerläßlich und demgemäß theoretisch — wie ja überhaupt die ,Ruhelage“" selbst — von großer Bedeutung. Das Prinzipielle sei hier mitgeteilt, betreffs genauerer Ausführung auf $A$. Tschermak ${ }^{6}$ ) und $M$. H. Fischer ${ }^{21}$ ) verwiesen.

Ein Beibbretthalter mit einem tadellos verfertigten, genau wagrecht gestellten BeiBbrett dient zur Fixation des Kopfes des Beobachters. Ein Auge wird mit einer schwarzen Binde vollkommen abgeschlossen, um die Faktoren der Fusion auszuschalten; dabei geht das abgedeckte Ange in Abblendungsstellung über. Allerdings ist hier zu berücksichtigen, da $B$ einige Zeit gewartet werden mu $B$ und dann noch immer ein Rest der durch die Fusionstendenz vor AbschluB des Auges hervorgerufenen Ausgleichsinnervation zurückbleiben kann, wie Hofmann und Bielschowsky ${ }^{14}$ ) nachgewiesen haben; erst nach längerer Zeit verschwindet diese Ausgleichsinnervation vollständig, doch ist ein minimaler Rest für unsere Untersuchungen von untergeordneter Bedeutung. Der Beobachter fixiert nun seinen Kopf und sucht mit Hilfe einer einmal wagrecht, das andere Mal senkrecht orien. tierten Nadel, die durch eine einfache Einrichtung verschiebbar ist, die objektiven Aquivalenzorte für den subjektiven Eindruckswert „Scheinbar Gleichboch“ und "Scheinbar Geradevorne" in verschiedenen Entfernungen auf; diese objektiven Aquivalenzorte werden in einem Koordinatensystem derart eingetragen, daß die Abszissen die Entfernungen vom Drehpunkte des Auges und die Ordinaten die Abweichungen vom objektiven Gleichhoch bzw. Geradevorne darstellen. Das objektive Gleichhoch und Geradevorne läßt sich leicht mit dem Tschermakschen Justiorblock*) finden.

Dadurch, daß dieses Verfahren mit jedem Einzelauge - natürlich immer unter vollständigem AbschluB des anderen - durchgeführt wird and die zugehörigen Werte nebeneinander eingetragen werden, ergeben sich Kurven für das scheinbare Gleichhoch und Geradevorne, aus deren Kombination sich ohne weiteres jene Stellungen für beide Einzelaugen für verschiedene Entfernungen finden lassen, welche den subjektiven Eindruckswerten scheinbar Gleichhoch und Geradevorne entsprechen. Nun ist es sehr wahrscheinlich, daß derjenigen Stellung des Einzelauges, welche uns beim Fernsehen und bei Mittelstellung des Kopfes - d. h. den Kopf bei aufrechter Körperhaltung gerade nach vorne gerichtet - den subjektiven Eindruck scheinbar Gleichhoch und Geradevorne vermittelt, ein Minimalspannungstonus sämtlicher Augenmuskeln entspricht, daß ihr das Minimalspannungsrezeptionsbild**) zu-

*) Siehe dessen Beschreibung bei Tschermatk ${ }^{7}$.

**) Wir schreiben nacb $A$. Tschermak'). S. 60,6), S. 40-4I den Augenmuskeln eine ganz charakteristische sensorjsche Bedeutung für die absolute Lokalisation zu, in der Weise, da.B die Muskelspanungen "sensible" Erregungen, nicht Empfindungskomponenten bedingen, deren Komplex eine relativ einfache Empfindung (z. B. geradevorn, gleichhoch) als psychischen Endeffekt hervorruft; die einzelnen Komponenten des objektiven Spannungsbildes der Augenmuskeln werden dabei nicht empfunden, die durch sie ausgelösten ,,sensiblen“ Erregungen besitzen keine BewuBtseinskorrelate. Es bedeutet also diese Auffassung, für welche sich neuerdings eine große Anzahl von Stützen finden ließen $\left[M . H\right.$. Fischer $\left.\left.{ }^{21}\right)\right]$, etwas Grundverschiedenes von der alten Lehre vom Stellungsbewußtsein. 
kommt; das bedeutet also, daß sie die "Ruhelage" in unserem Simne, die "tonische" Gleichgewichtslage darstellt. Dagegen wird sofort eingewendet werden, $\mathrm{da} B$ es doch unter diesen Umständen nicht möglich ist, die willkürlichen Innervationen und Augenbewegungen vollständig auszuschalten. Dem ist folgendes entgegenzuhalten. Verfasser beschäftigte sich mit diesem Probleme fast ununterbrochen durch 2 Jahre, und immer wieder ergab sich bei seinen wiederholten Untersuchungen, daß der Eindruck Scheinbar-Gleichhoch bzw. Scheinbar-Geradevorne eine ganz bestimmte Augenstellung verlangt, die mit größter Bestimmtheit. dann aufzufinden ist, wenn gar nicht eine spezielle Aufmerksamkeit auf die Einstellungen verwendet wird, die Augen vielmehr sich selbst überlassen werden. Trotz absichtlicher willkürlicher Augenbewegungen und trotz Fehlens jeglicher Anhaltspunkte wird - mit Ausnahme gering. fügiger Fehler und Schwankungen, wie sie sich ja nie vermeiden lassen - in einer Versuchsreihe immer wieder dieselbe Stellung herausgefunden, wenn die Einstellungen sich nicht über eine allzulange Zeit hinaus erstrecken und infolge Ermüdung eine allzugroße Schwankungsbreite ergeben, was natürlich durch Abbrechen der Experimente vermieden werden kann. Alle subjektiven Untersuchungsmethoden erlauben ja überhaupt nur eine relativ kurze Arbeitszeit, wenn sie exalkte Resultate liefern sollen; zumindest müssen größere Ruhepausen eingeschoben werden. Es ist also sicher, daß die oben charakterisierte Augenstellung durch etwas ganz Bestimmtes ausgezeichnet ist, sonst wäre ihre Konstanz unverständlich, und es liegt nahe, sie als durch den Minimalspannungstonus sämtlicher Augenmuskeln ausgezeichnet zu betrachten. Daß die „Ruhelage" zeitlichen Schwankungen unterliegt, wie vom Verfasser gleichfalls schon nachgewiesen wurde, darf uns nicht wundernehmen und spricht ganz und gar nicht gegen unsere Auffassung, denn es ist doch offenbar nicht zu erwarten, daß die Verteilung des ,Tonus" immer dieselbe bleibt, sondern vielmehr gewissen Schwankungen unterliegt*). Mit der besprochenen Methodik ergab sich damals bei einer Beobachtungsdistanz von $130 \mathrm{~cm}$ bzw. einer Akkommodation von $0,77 \mathrm{D}$ nach Vollkorrektur (r. A. + 0,75, 1. A. - 1) als „Ruhelage" der beiden Einzelaugen für mich eine Rechtswendung des rechten Auges von etwa $6^{\circ} 40^{\prime}$, eine Linkswendung des linken Auges von etwa $3^{\circ} 53^{\prime}$, ferner eine Senkung beider Angen von im Mittel $3^{\circ} 26^{\prime}$ (stärker am linken Auge), also eine absolute Divergenz und eine Senkung, außerdem eine bestimmte Rollung im Sinne einer Divergenz der Längsmittelschnitte nach oben, von weleher noch später Genaueres auszuführen sein wird. Für A. Tschermak ${ }^{6}$ ), allerdings einen schielenden Myopen mit anomaler Sehrichtungsgemeinschaft, besteht jenseits des Fernpunktes die „Ruhelage" beider Einzelaugen in einer absoluten. Divergenz von etwa $50^{\prime}$ ind Rechtswendung

*) Davon wird noch an anderer Stelle Näheres berichtet werden. 
des rechten Auges von $1^{\circ} 36^{\prime}$, des linken Auges von $45^{\prime} 50^{\prime \prime}$, ferner in Senkung von $3^{\circ} 49^{\prime} \mathrm{im}$ Mittel (rechtes Auge stärker als linkes); im Schielfalle Krause (bei T'schermak) zeigten die „Ruhelagen“ jenseits des Fernpunktes eine 'Konvergenz von etwa $5^{\circ} 34^{\prime}$ ohne ausgesprochene Seitenwendung. - Auch diese Befunde sprechen für unsere Auffassung; fanden ja doch auch die früher genannten Autoren in der Mehrzahl der Fälle eine Divergenz der Gesichtslinien bei ihren Untersuchungen über die anatomische Ruhestellung, die - wie bereits eingangs erwähnt - keine großen Differenzen gegen die funktionelle „Ruhelage" aufweisen dürfte. Kinematische Primärstellung und "Ruhelage" sind also bei mir inkongruent, und es ist zu erwarten - was noch zu begründen sein wird -, daß ein solches Verhalten auch bei normalen binokularsehenden Isoemmetropen die Regel ist*).

\section{Die „Abblendungsstellung“ der Augen.}

\section{A. Geschichte und Untersuchungsmethodik.}

Die Abweichung eines abgedeekten Auges von der Fixationsstellung unter Beibehaltung der Fixation des anderen (von Stevens Heterophorie genannt) bildete aus naheliegenden Gründen schon seit langem den Gegenstand vielfacher Untersuchungen.

Die objektive Schätzung des Ablenkungswinkels unter der deckenden Hand wurde als zu grob und unzuverlässig bald fallen gelassen und durch eine Anzahl exakterer, subjektiver Methoden ersetzt. Es sei hier das Verfahren Albrecht v. Graefes erwähnt, die Fusion durch Vorsetzen eines vertikal ablenkenden Prismas auszuschalten und die Heterophorie aus der Distanz der auftretenden Doppel bilder zu berechnen, welches wohl heute infolge der ihm anhaftenden mannigfachen Fehlerquellen kaum mehr Verwendung finden dürfte. E. $E$. Maddox ${ }^{25}$ ), spez. S. 221-244, ersetzte das Prisma zuerst durch ein Doppelprisma mit horizontaler Kante, dann durch ein Glasstäbchen und schließlich durch seinen allgemein bekannten, zumeist aus rotem Glase zwecks farbiger Differenzierung der Doppelbilder verfertigten Glasstäbcheneinsa tz. Durch Kombination mit seiner Tangentenskala - ähnliche Tangentenskalen waren bereits von Landolt und Hirsch-

*) Über die spezielle Bedeutung meiner früheren Untersuchungen [M. $H$. Fischer ${ }^{21}$ )] betreffs der Abhängigkeit der Augenstellung (der "Tonus" =Verteilung an den Augenmuskeln) vom Labyrinthe und der Halsmuskulatur am Menschen wird Genaueres in einer folgenden Arbeit auszuführen sein; es sei hier nur auf die interessante Tatsache hingewiesen, da.B meine Resultate weitgehende Übereinstimmung mit den von $R$. Magnus und seiner Schule an Katzen und Kaninchen gewonnenen Ergebnissen erkennen lassen. Das konnte seinerzeit noch nicht genügend berücksichtigt werden, weil eine Anzahl der einschlägigen Arbeiten noch nicht vorlag. Jedenfalls ist damit wieder einmal erwiesen, welche Bedeutung der exakt-subjektivistischen Untersuchungsmethodil zuzuschreiben ist, da sie Ergebnisse zeitigt, zu denen objektive Prüfungen oft wertvolle Parallelen liefern und umgekehrt. Ganz ähnliche Parallelen auf dem Gebiete der Labyrinthphysiologie werden von uns später beschrieben werden. 
berg angegeben - war damit eine einfache Methode zur Untersuchung der Heterophorien gegeben, welche auch allseitig verwendet wird. Betreffs der zum Teil allerdings sehr gewichtigen Einwände, die sich gegen dieses Verfahren erheben lassen [vgl. speziell $A$. Tschermak und Schlodtmann ${ }^{22}$ )], sei auf einen folgenden Abschnitt verwiesen.

Unsere systematischen Untersuchungen wurden an einem ausgewählten Studentenmateriale mit einer von $A$. Tschermak angegebenen Apparatur ausgeführt, wie ich sie bereits früher beschrieben habe [M. H. Fischer $\left.\left.{ }^{21}\right)\right]$. Es sei ihrer Wichtigkeit halber gestattet, sie hier nochmals in kurzen Worten an Hand einer leicht faßlichen Abbildung zu schildern. Der Vorteil dieser Methode ist ihre Exaktheit*), wie sie bisher noch mit keiner anderen erreicht werden konnte, dabei größtmöglichste Einfachheit und leichte, rasche praktische Verwertbarkeit;

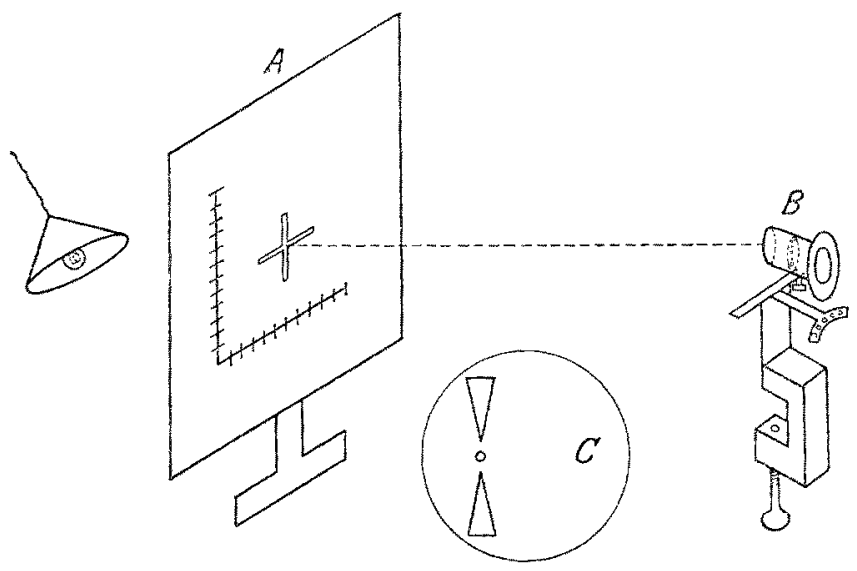

Abb. 1.

schließlich ist der dazu nötige Apparat so wohlfeil, daß er leicht eine verbreitete Anwendung finden könnte. Allerdings ist die Methode nur dann verwertbar, wenn beide Augen sehtüchtig sind.

Die Einrichtung besteht im wesentlichen aus folgendem (Abb. 1): In einem mit schwarzem Papier uberzogenen Rahmen $A$ wird ein rechtwinkliges, $2 \mathrm{~cm}$ langes und $2 \mathrm{~cm}$ breites Kreuz mit etwa 2-3 mm Spaltbreite eingeschnitten, das von rückwärts durch eine Glühlampe hell erleuchtet ist; parallel zum horizontalen und vertikalen Schenkel ist in einiger Entfernung eine Millimeterskala aufgeklebt. In bestimmter Entfernung von $A$ wird ein Beißbrett zur Fixation des Kopfes des Beobachters - welohe eine nnerläBliche Bedingung bei allen exakten Bestimmungen ist - festgeschraubt. Der BeiBbretthalter trägt einen Querbalken mit einem angeschraubten photographischen Momentverschluß, der gegen das Auge des Beobacbters hin durch eine Röhre ans schwarzem, lichtdichtem Stoffe verlängert ist. Der MomentverschluB ist nach rechts und links verschiebbar, die Stoffröhre

*) Es lassen sich auch Rollungsabweichungen mit ihr bestimmen. 
kann an das jeweilig benutzte Auge lichtdicht angepaßt werden. Die vordere Öffnung des Momentverschlusses ist durch eine schwarze Kreisscheibe mit einem kleinen kreisförmigen und zwei exzentrischen keilförmigen Abschnitien (Abb. $1 C$ ) abgedeckt; die beiden keilförmigen Ausschnitte, welehe nur die Aufgabe haben, den Eindruck des abblendbaren Auges speziell zu kennzeichnen, werden mit einer dünnen Schicht farbiger Gelatine überzogen. Beobachter fixiert seinen Kopf durch Eimbeißen, stellt dann den geöffneten Verschluß nach Anpassen der Stoffröhre an das eine Auge so, daß er mit diesem durch den kleinen kreisförmigen Scheibenausschnitt und gleichzeitig mit dem anderen freien Auge das hellerleuchtete Kreuz fixieren kann, dieses also binokular sieht. Es wird also Binokularsichtbarkeit der Fixiermarke mit unokularer Kennzeichnung des einen temporär abgeblendeten Auges kombiniert. Der Verschluß kann nach Belieben geschlossen und wieder geöffnet werden*).

Es sei erlaubt, einiges zur Nomenklatur zu bemerken. Orthophorie ist dann gegeben, wenn beim Fernesehen die Blicklinien bei Ausschluß aller nervösen Einflüsse, oder besser aller Fusionsfaktoren parallel und geradeaus gerichtet sind, die Vertikalmeridiane einander parallel laufen $\left[\right.$ Bielschowsky $\left.\left.{ }^{12,13}\right)\right]$; alle anderen unter denselben Bedingungen vorhandenen Stellungen der Blicklinien und Vertikalmeridiane werden als Heterophorien bezeichnet. Bielschowsky ${ }^{12,13}$ ) teilt dieselben in scheinbare, d.h. auf nervöser Grundlage beruhende und wirkliche, durch mechanische (anatomische und topographische) Faktoren bedingte ein, eine Einteilung, der wir uns nicht anschlieBen können, da wir die „Ruhelage" als eine ,tonische", funktionelle auffassen müssen, welche ohne nervöse Beeinflussung der Augenmuskeln nicht denkbar ist. Klar und eindeutig sind auch die Begriffe Esophorie im Sinne von Einwärtsabweichung, des nicht-fixierenden Auges und Exophorie im Sinne von AuBenabweichung des nicht-fixierenden Auges. Bei Vertikaldivergenzen wäre es zweckmäßig, neben den allgemein bekannten Benennungen Hyperphorie (sursum vergens) und Hypophorie (deorsum vergens) auch an die einfachere Bezeichnung $E$. Herings zu denken, der unter positiver Vertikaldivergenz eine Abweichung des rechten Auges nach

*) Unserer Versuchsanordnung ähnlich, aber praktisch schwer verwendbar, ist die Maddox sche Sehkammer [Maddox-Asher ${ }^{25}$ ), S. 274-281]. Neuerlich wurde von Baldino ${ }^{25}$ ) ein Apparat nach dem Prinzip von Cantonnet (Arch. d'ophthalmol. Juni 1918 und Nov./Dez. 1918) konstruiert; derselbe besteht aus zwei geschwärzten verschieblichen Röhren; die eine offene wird vor das eine, die andere mit durchscheinendem Papier, in dessen Mitte sich ein kleines Loch befindet, geschlossene wird vor das andere Auge gebracht; auch dieser Apparat ersoheint unhandlich. Sidney P. Holloway (The maddox ,wing test" for heterophoria: ophthalmoscope $12,342-343$. 1914) stellt vor beide Augen je eine Blendenöffnung und in Leseentfernung dazu eine Skala mit einem senkrecht dazu gestellten Pfeile. Durch ein System von zwei Klappen, welches sich zwischen den Blenden and der Sehprobe befindet, wird von der Zeichnung die eine Hälfte nur dem einen Auge und die andere Hälfte nur dem andéren Auge geboten, so daB aus der Stellung des Pfeiles zu der Skala auf die Lage der Augen in der entsprechenden Entfernung geschlossen werden kann. 
oben bzw. des linken Auges nach unten bei fixierendem andern versteht, unter negativer Vertikaldivergenz das umgekehrte. Betreffs der als Cyclophorie bezeichneten Rollungsabweichungen fehlt eine einheitliche detailierte Nomenklatur. Die $E$. Heringschen*) Bezeichnungen positive Rollung, d. h. von hinten gesehen eine Rollung um die Gesichtslinie im Sinne des Uhrzeigers oder um die hintere bzw. retinopolare**) Halbachse und negative Rollung, $d$. h. eine Rollung entgegengesetzt dem Sinne des Uhrzeigers oder um die vordere bzw. corneopolare**) Halbachse beziehen sich immer auf eine gleichmäßige Rollung beider Augen, wie sie beispielsweise reflektorisch von seiten des Labyrinthes bzw. der Halsmuskeln ausgelöst werden kann. Wir möchten für die Rollungsabweichungen folgende Nomenklatur als zweckmäßig vorschlagen:

Als positive Rollungsabweichung sei eine solche bezeichnet, welche von hinten gesehen - mit einer Drehung des rechten Auges um die Gesichtslinie im Sinne des Uhrzeigers, also mit dem oberen Pole nach außen oder um die hintere bzw. retinopolare Halbachse, oder mit einer Drehung des linken Auges um die Gesichtslinie entgegengesetzt dem Sinne des Uhrzeigers, also gleichfalls mit dem oberen Pole nach außen oder um die vordere bzw. corneopolare Halbachse verlnnüpft ist, wobei jedesmal das andere Auge als fixierend gedacht ist. Bei der Betrachtung von vorne ist eine positive Rollungsabweichung charakterisiert durch eine Drehung des rechten Auges entgegengesetzt dem Sinne des Uhrzeigers mit dem oberen Pole nach außen (um die hintere bzw. retinopolare Halbachse) oder durch eine Drehung des linken Auges um die Gesichtslinie im Sinne des Uhrzeigers mit dem oberen Pole nach außen (um die vordere bzw. corneopolare Halbachse). Es besteht sonach Divergenz der Längsmittelschnitte nach oben. - Eine negative Rollungsabweichung bedeutet dann das gerade umgekehrte Verbalten. Die Rollungsabweichungen erscheinen also durch folgende Einzelgruppen charakterisiert.

1. Positive Rollungsabweichungen.

a) Linkes Auge fixiorend.

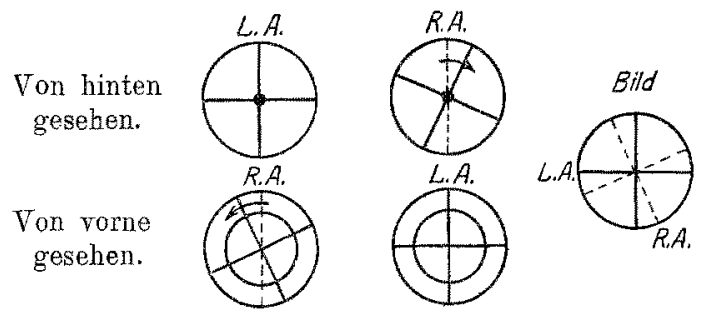

Abb. 2.

*) E. Hering ${ }^{1}$, S. $\left.489 ;{ }^{9}\right)$, \$. 48 .

**) Diese bei Hering nicht gebrauchten Bezeichnungen seien aus praktischen Gründen eingeführt. 
b) Rechtes Auge fixierend.

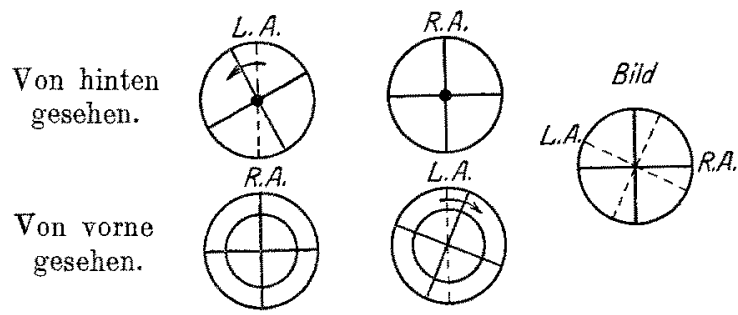

Abb. 3.

2. Negative Rollungsabweichungen.

a) Linkes Auge fixierend.

Von hinten
gesehen.

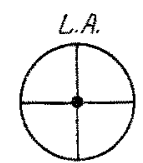

Von rome gesehen.
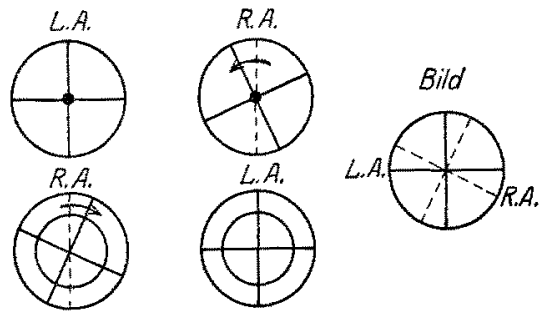

Abb. 4.

b) Rechtes Auge fixierend.

Von hinten gesehen.
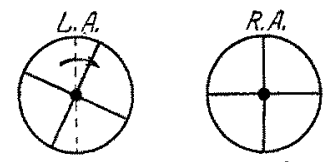

Von vorne
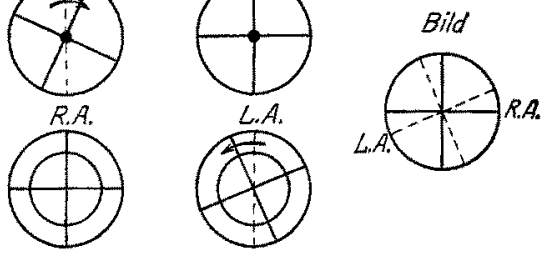
gesehen.

Abb. 5 .

\section{B. Ergebnisse eigener Beobachtungen.}

\section{a) Umfassende Beobachtungsreihe.}

Unsere Untersuchungen - 50 an Zahl - wurden an einem intelligenten Studentenmateriale der jüngeren medizinischen Studiensemester und einzelnen Ärzten ausgeführt; sämtliche waren normale Binokularsehende, die Mehrzahl Emmetrope, der Rest verteilte sich auf Myope und vereinzelte Hypermetrope. Es erwies sich als wichtig, den einzelnen Versuchspersonen vor dex Prüfung nur die allernötigsten Informationen zu erteilen. Denn es gibt - wie ja hinlänglich bekannt [F.B. Hofmann und A. Bielschowsky $\left.\left.{ }^{14}\right)\right]$ - eine große Anzahl von Menschen, unter ihnen 
besonders solche, welche sich häufig unokular beschäftigen und dabei das andere Augen offen lassen, also z. B. Mikroskopiker, Spektroskopiker, Jäger usw., schließlich auch geübte freiäugige Stereoskopiker, die infolge Übung willkürlich entgegen jedem Fusionszwange konvergieren evtl. auch divergieren können, ja dieses selbst vermögen, wenn ein Auge geschlossen bzw. abgedeckt ist. Bei unseren Untersuchungen müssen aber Willkürmotive ausgeschaltet sein. Übrigens machen solche Versuchspe sonen - bei mir in 2 Fällen - immer selbst darauf aufmerksam, daß sie imstande sind, die horizontale Distanz der bei unseren Versuchen auftretenden Doppelbilder innerhalb bestimmter Grenzen willkürlich zu mehren und zu mindern.

Bei unseren Untersuchungen wurde in folgender Weise vorgegangen: Jeder Beobachter verfertigte sich ein BeiBbrett; nachdem dasselbe am beschriebenen Hal. ter angeschraubt worden war, fixierte die Versuchsperson durch Finbeißen ihren Kopf, die Stoffröhre wurde an ein Auge angepa Bt und der Verschluß geöffnet. In verlangter Distanz wurde parallel zur Frontalebene der Rahmen mit dem durch leuchteten Kreuze so gestellt, daß der Beobachtex mit dem einen Auge durch den kreisförmigen Ausschnitt der Blende im Verschlusse, mit dem anderen frei das leuchtende Kreuz sehen konnte, es also binokular dargeboten erhielt. Nachdem er angewiesen war, mit dem freien Auge da uernd zu fixieren, schloß der Versuchsleiter den Verschluß vor dem temporär abzublendenden Auge und offnete ihn nach einiger Zeit wieder für Momente von $1 / 50-1$ Sekunde, evtl. auch Iänger. Betreff der. Öffnungsdauer mußte, wie sich gleich anfangs erga $b$, individualisiert werden, je nachdem die Fusion bei den einzelnen Versuchspersonen ausgeprägt war und ihnen die Fähigkeit zukam, die auftretenden Erscheinungen - Doppelbilder in charakteristischer Seiten-, Höhendistanz und eventueller Neigung zueinander - im Detail aufzufassen.

Es sei gleich hier bemerkt, daß sich sichere Anhaltspunkte anführen lassen, die eine prinzipielle Scheidung zwischen Fusionsbreite und Fusionsanspruchsfähigkeit verlangen. Es gibt Individuen, die eine große Fusionsbreite besitzen, bei denen aber die Ausgleichsinnervation nur ungemein langsam bei Freigabe des verdeckten Auges einsetzt und. sehr träge verläuft, bei denen also die Fusionsanspruchsfähigkeit eine geringe ist*). Umgekehrt fanden sich Beobachter, bei denen überhaupt. jede Ausgleichsinnervation ausblieb, wenn die Distanz der bei Freigabe des bisher verdeckten, abgewichenen Auges auftretenden Doppelbilder einen. bestimmten relativ kleinen Wert überschritten hatte, bei denen also die Fusionsbreite relativ gering war; unterschritt aber die Distanz der Doppelbilder diesen Grenzwert, trat die Ausgleichsinnervation so rasch

*) Solche Individuen vermochten die bei Öffnung des Verschlusses infolge des. unwillkürlichen langsamen Einstellungsaktes des bisher abgewichenen Auges auftretenden gegensätzlichen Scheinbewegungen der Sehdinge (des leuchtenden Kreuzes) sehr genau zu beobachten und zu beschreiben. - Bei altivem LidschluB fliehen beide Augen nach oben (Bell sches Phänomen); öffnet man die Augen, so sieht man bei einiger Aufmerksamkeit eine Scheinbewegung der Sehdinge nach oben. 
in Aktion, daß es vielen der Beobachter erst nach einiger Utbung möglich war, anzugeben, daß im ersten Momente überhaupt Doppelbilder vorhanden waren*).

Gegen die Maddox sche Methode ist der Einwand zu erheben, daB sie durch Vorsetzen eines farbigen (roten) Glasstäbchensatzes unter Beschränkung beider Augen auf bloß unokulare Eindrücke, also unter dauernder, jedoch verschiedenartiger Beanspruchung jedes Einzelauges die Abblendungsstellung herbeiführt; prinzipiell richtig ist nur ein vollständiger Ausschluß des einen Auges vom Sehakte unter Vergleich mit. der zuvor wie nachher erfolgenden zweiäugigen Darbietung des Fixationsobjektes (neben den unokularen Differenzierungsmarken des geprüften Auges). Es steht fest, da $B$ die Abbildungsverhältnisse die relative Stellung beider Augen und damit die Abblendungsstellung zu beeinflussen imstande sind, und zwar in jedem Einzelfalle in charakteristischer Weise, wie bereits $T$ schermak ${ }^{6}$ ) und Schlodtmann ${ }^{22}$ ) in exakter Weise ausgeführt haben und dies neuerdings wieder von mir $^{21}$ ) gezeigt wurde. Es ist sicher, daß vollständige Abdeckung eines Auges in einer gxößeren Zahl Heterophorie hervortreten läßt (bes. bei Fällen der 2. Gruppe mit ausgesprochener Fusionsanspruchsfähigkeit), als die Untersuchung mit dem Maddox schen Glasstäbchensatz erkennen läßt. Dies und überhaupt die Genauigkeit unserer Methode lassen es verstehen, daß wir weitaus weniger Orthophorien (eine vollkommen exakte überhaupt nicht!) fanden als Bielschowsk $y^{54}$ ), der, wenn auch mit einem gewissen Vorbehalt, aus seinen Befunden schließt, daß es bei normalen Binokularsehenden höchstens $20-25 \%$ Orthophorien gibt**).

Als Paradigma unserer Untersuchungen sei der Befund bei dem emmetropen, normal binokularsehenden S. H. (23 Jahre alt) gegeben (s. Abb. 6 u. 7, S. 266):

Es ergibt sich hier in $1 \mathrm{~m}$ Entfernung eine nicht symmetrische Exophorie für beide Augen (für das R.A. von $55^{\prime}$, für das L.A. von $1^{\circ} 9^{\prime}$ ), eine negative Vertikaldivergenz für beide Augen (für das R.A. also eine Hypophorie von etwa 14', für das L.A. eine geringe Hyperphorie) und für beide Augen eine mäßige positive Rollungsabweichung ( $\mathrm{d} . \mathrm{h}$. für das R.A. eine Rollung um die Gesichtslinie im Sinne des Uhrzeigers mit dem oberen Pole nach außen, für das L.A. eine Rollung

*) Bei diesen Versuchspersonen konnte - wenn die relativ geringe Fusionsbreite überschritten war — der Verschluß dauernd geöffnet bleiben, ohne daß sich an den Doppelbildern irgend etwas änderte; als interessant ist zu exwähnen, da $B$ hie und da sogar die Doppelbilderdistanz allmählich zunahm, eine Erscheinung, die man als eine anpassungsmäBige auffassen kann, indem dadurch das Halbbild des abgewichenen Auges - entsprechend der Abnahme der Bild-und noch mehr der Sehschärfe im indirekten Sehen - objektiv wie subjektiv unschärfer bzw. weniger eindringlich wird und leichter der inneren Hemmung unterliegt.

**) Neuerdings schreibt Bielschowsky ${ }^{23}$ ) 8.166 allerdings schon, ,wir wissen. nicht nur, da $B$ fast jeder Mensch eine Heterophorie hat usw.", an einer anderen Stelle, S. 191, ,eine ideale anatomische Ruhelage (Orthophorie) ... kommt höchstens ganz ausnahrosweise vor". 
um die Gesichtslinie entgegen dem Sinne des Uhrzeigers gleichfalls mit dem oberen Pole nach außen, immer in der Ansicht von hinten*).

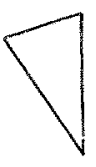<smiles>[R4]C(C)(C)C</smiles>
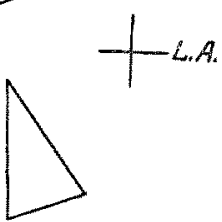

Abb. 6. Aluftretende Doppelbilder**) bei momentaner Freigabe bilder bes momentanor des ca. $2^{\prime}$ unter Verschluß ge- gabe des ca. $2^{\prime \prime}$ unter Versehluß haltenen reohten Auges (R.A.O) gehaltenen linken Auges (L. in $1 \mathrm{~m}$ Distanz, bei fixierendem A. e) in $1 \mathrm{~m}$ Distanz, bei fixielinken Ange. rendem rechten Auge.

Die Berechnung gestaltet sich folgendermaßen:

a) für de tatsüchiche Augen* stellung (Abb. 8)

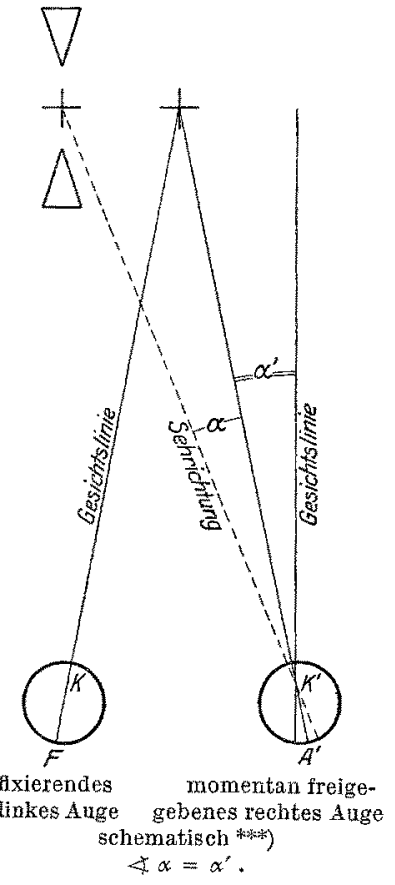

Abb. 8 . b) auf cas Doppelauge bezogen (Abb. 9)

$\sqrt{7}$

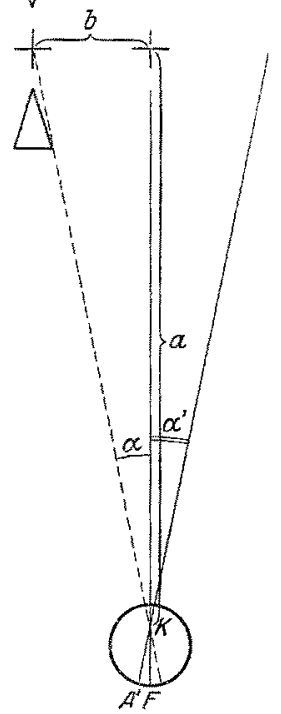

schematisch

$\varangle \alpha=x^{\prime} ;$

Abb. 9. $\operatorname{tg} \alpha=\operatorname{tg} a^{\prime}=\frac{b+)}{a}$.

*) Bei meinen Selbstbeoba chtungen bemerkteich (meine Fusionsanspruchsfähigkeit ist relativ gering - ich kanni die Doppelbilder lange festhalten -), da $B$ nach Aufgabe der Fixation des bisher fixierenden Auges bei geöffnetem Verschlusse vor dem anderen Auge, dieses die Fixation übernehmen konnte, ja sogar der Blick beliebig wandern konnte, ohne da B eine Anderung der Doppelbilderdistanz auftrat und daB die Doppelbilder fix an Ort und Stelle zu bleiben schienen; ich war anfangs geneigt, zu glauben, da $B$ hier eine reine Auf. merksamikeitswanderung ohne Veränderung der Aagenstellung im Spiele sei, bis ich mich an Prof. R. $Z$. Kahm, der dasselbe Phänomen beobachitete, iiberzeugen konnte, da $B$ die Augen tatsächlich Bewe. gangen ausführten. Es kann also die relative Lage der Augen zueinander trotz Blickwonderung dieselbebleiben!

**) Dieser Fall wäre also - wenn auch nicht ganz streng - zu den alternierenden Hyperphorien zu rechnen.

****) Wegen der prinzipiellen Inkommensurabilität der Winkel der objektiven Richtungslinien. und der subjektiven Sehrichtungen!

†) $a$ ist die Entfernung des beobachteten Kreuzes vom Knotenpunkte des Auges (ungefähr äußerer Augenwinkel), $b$ die direkt meBbare Distanz der Doypelbilder. 
In derselben. Weise wie es hier für Breitenabweichungen ausgeführt ist, lassen sich die Höhenabweichungen berechnen, die Rollungsabweichungen sind direkt meBbar.

Eine detaillierte Übersicht über die untersuchten 50 Fälle in Form einer Tabelle sei infolge der zu großen Ausdehnung derselben unterlassen und nur eine Zusammenfassung in Gruppen geboten.

Unter 29 Ermmetropen waren 4 fast vollkommene Orthophorien und 25 Heterophorien:

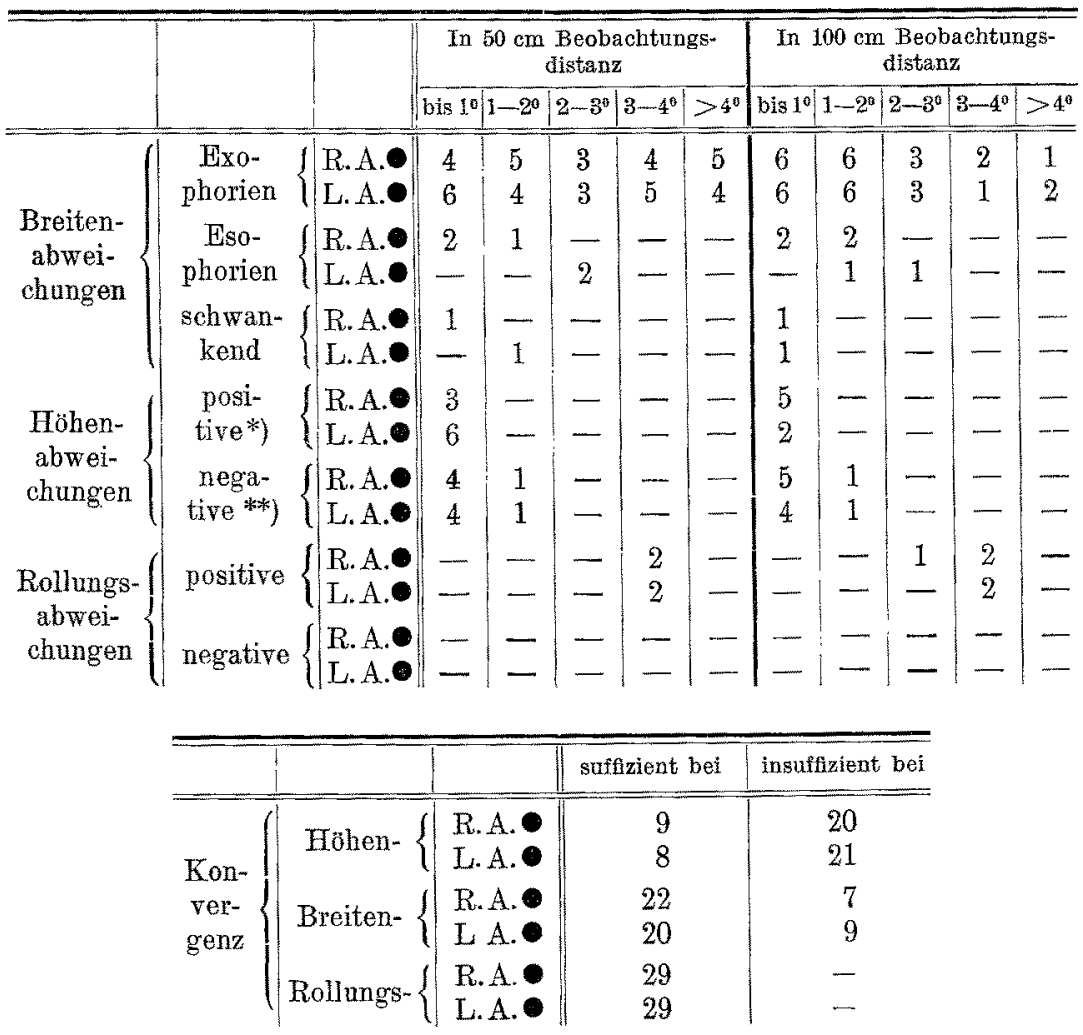

Von den 29 Emmetropen waren:

\begin{tabular}{c|c|c|c}
\hline $\begin{array}{c}25 \text { Heterophorien } \\
\text { nicht } \\
\text { alternierend }\end{array}$ & symmetrisch & asymmetrisch \\
\hline 24 & 1 & 3 & 22
\end{tabular}

*) D. h. also Hyperphorie des R. A. bzw. Hypophorie des L. A.

**) Gerade das Umgekehrte. 
Bei den 29 Emmetropen*) war die Fusionsanspruchsfähigkeit**):

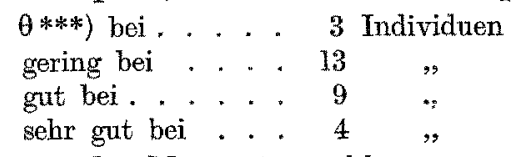

Nach Daueröffnung des Momentverschlusses vor dem abgedeckten Auge

blieb die Doppelbilderdistanz gleich bei .......4 4†) Individuen

verminderte sie sich bui . . . . . . . . . 7 " ",

die Doppelbilder verschwanden bei . . . . . . 18 "

Unter 21 Refraktionsanomalen $\dagger^{\dagger}$ ) war keine angenäherte Orthophorie, sondern durchweg Heterophorien $\left.\dagger_{\dagger} \dagger\right)$ folgenden Charakters:

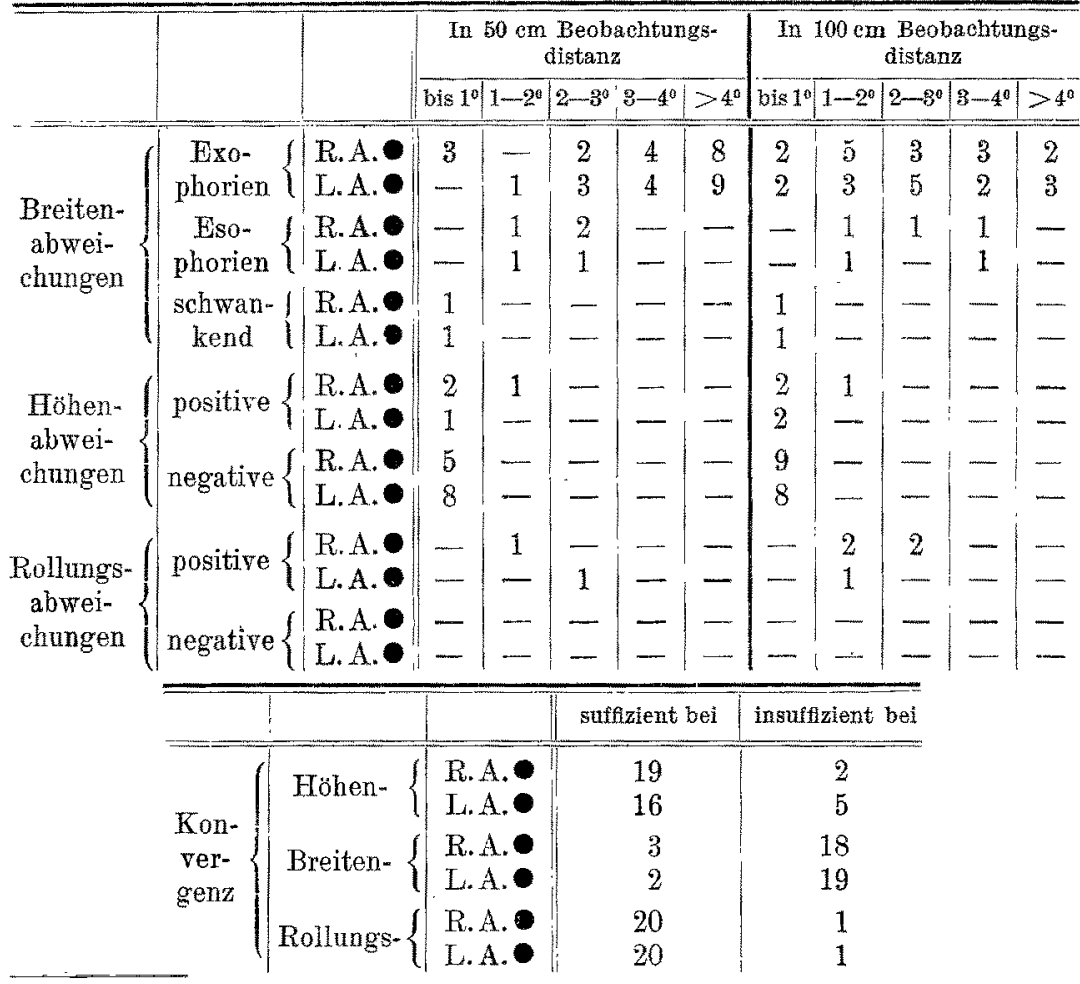

*) Hier sind auch die vier oben genannten Orthophoren mit eingerechnet, weil anch sie keine ideale Orthophorie zeigten, sondern nur ganz minimale Abweichungen, die infolge der ausgezeichneten Fusionsanspruchsfähigkeit dieser Individuen nur bei Momentöffnung $\left(1 / 2{ }^{\prime \prime}\right)$ nachweisbar waren, sonst sofort verschwanden.

**) Über die Fusionsbreite läßt sich natürlich aus unseren Versuchen im allgemeinen nichts aussagen.

***) Unter den gewählten Beobachtungsbedingungen.

†) Bei diesen vier Versuchspersonen mit relativ geringer Fusionsbreite war bei einem von ihnen nur zeitweise -- offenbar die Fusionsbreite bereits iberschrit- 
Von 21 Refraktionsanomalen waren:

\begin{tabular}{c|c|c|c}
\hline \multicolumn{2}{c|}{$\begin{array}{c}\text { 21 Heterophorien } \\
\text { nicht } \\
\text { alternierend }\end{array}$} & 2ymmetrisch & asymmetrisch \\
\hline 21 & 0 & 4 & 17
\end{tabular}

Bei den 21 Refraktionsanomalen war die Fusionsanspruchsfähigkeit:

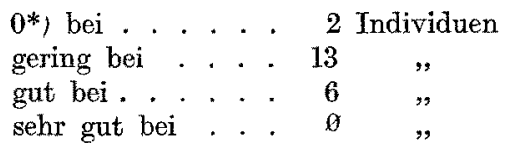

Nach Daueröffnung des Momentverschlusses vor dem abgedeckten Auge

blieb die Distanz der Doppelbilder gleich bei. . . . . 2 Individuen

verminderte sie sich bei .......... 11 ",

die Doppelbilder verschwanden bei . . . . . . 8 "

Man wird gleich bei Durchsicht obiger Resultate den Einwand ererheben, da $\beta$ die Heterophorieuntersuchungen bei Distanzen von 50 und $100 \mathrm{~cm}$, also beim Nahesehen angestellt wurden, wo doch zu scheiden ist zwischen Heterophorie beim Fernesehen und Heterophorie beim

ten, so da B eine binokulare Einstellung nicht erfolgen konnte. Zwei davon zeigten sogar eine allmähliche Zunahme der Doppelbilderdistanz, eine adaptative Erscheinung, die - wie bereits bemerkt - dazu führt, das eine Bild immer mehr in die Netzhautperipherie hinauszudrängen und damit dessen Schärfe und Eindringlichkeit zu schwächen. Dasselbe Phänomen tritt übrigens - wie ja allgemein bekannt - bei manifest Schielenden relativ häufig auf. Der Unterschied den Schielenden gegenüber besteht nur darin, daß nach vollständiger Freigabe der abgeblendeten Augen durch aktiven LidschluB und Augenbewegungen bei allen Individuen normales Binokularsehen auftritt. Die Versuchspersonen werden gewissermaßen nur unter den gegebenen Bedingungen zu „Anomalen", manifest Schielenden, während sie sonst ein völlig normales Verhalten zeigen. Die Methode läßt also eine verfeinerte Auslese unter den sonst normalen Binokularsehenden $z u$, denn es ist zweifellos, da $B$ durch die charakteristisehe Abblendung des einen Auges selbst bei geöffnetem Verschlusse - das Sehfeld dieses Auges als auslösendes Moment für den Fusionsakt ist doch relativ klein - eine Minderung sowohl der Fusionsanspruchsfähigkeit als auch der Fusionsbreite bewirkt wird. Willkürakte müssen natürlich bei diesen Prüfungen durchaus ausgeschaltet sein!

t†) Davon waren 19 Myope bis zu 8 Dioptrien, 2 Hypermetrope bis zu 3 Dioptrien; 5 waren anisometrop, 2 presbyop. Sämtliche Untersuchungen wurden naoh Vollkorrektur vorgenommen. Ein Hypermetroper (bds. +3) zeigte ohne Glas für das R. A. - bei $50 \mathrm{~cm}$ eine Exophorie von $9^{\circ} 26^{\prime}$, bei $100 \mathrm{~cm}$ $4^{\circ} 7^{\prime}$, für das L. A. $7^{\circ} 58^{\prime}$ und $4^{\circ}$, mit Vollkorrektur eine erhebliche Verminderung derselben, für das R. A. $6^{\circ} 44^{\prime}$ und $4^{\circ}$, für das L. A. $6^{\circ} 23^{\prime}$ und $2^{\circ} 18^{\prime}$.

††† Einer von diesen Fällen war nur bei $50 \mathrm{~cm}$ Beobachtungsdistanz exophorisch, bei $100 \mathrm{~cm}$ fast vollkommen orthophorisch; es handelt sich also bei ihm nur um Insuffizienz des Konvergenzapparates.

*) D. h. unter den gegebenen Beobachtungsbedingungen. 
Nahesehen, wobei in der letzteren immer Insuffizienzen des Näherungsapparates darinstecken. Ein solcher Einwand erscheint gewiß berechtigt, und es wäre sicherlich wünschenswert gewesen, die Prüfungen noch auf Distanzen von $2,3,4,5$ und $6 \mathrm{~m}$ auszudehenen, - von einigen wird Verfasser noch später berichten, speziell über eine große Anzahl zeitlich auseinanderliegender Eigenbeobachtungen, die uns manches Neue und Interessante bringen werden - doch ist es sehr zeitraubend (eine ganze exakte Reihe dauert 2-3 Stunden bei einer Versuchsperson!) und soll einer anderen Seite zur Vervollständigung empfohlen werden; die Wichtigkeit für die Praxis ist ja unverkennbar. Úbrigens zeigen $50 \%$ der Versuchspersonen (25) bei Zunahme des Konvergenzhalbwinkels um fast $2^{\circ}$ zwischen 100 und $50 \mathrm{~cm}$ Distanz eine fast völlige Suffizienz wenigstens der Breitenkonvergenz; es ist daher nicht zu erwarten, da 3 bei $\mathrm{Ab}$ nahme des Konvergenzwinkels dieselbe insuffizient werden sollte. Die restlichen $50 \%$ mit Breitenkonvergenzinsuffizienz fallen zum größten Teile den Refraktionsanomalen zur Last [trotz Vollkorrektur!*)]. Die Rollungskorrektur auf angenäherten Parallelismus bzw. mäßige symmetrische Divergenz der Längsmittelschnitte nach oben erwies sich nur in einem Falle als insuffizient. Die Höhenkorrektur auf Einstellung der Quermittelschnitte in eine Ebene war in ungefähr $50 \%$ suffizient (die weitaus größere Zahl stellen die Refraktionsanomalen), in $50 \%$ insuffizient (in der Mehrzahl Emmetrope). Es ist durchaus nicht notwendig, da $B$ sich beide Augen symmetrisch verhalten**), es ist das im Gegenteil das viel seltenere Vorkommen. Von den 46 Hoterophorien $[92 \% * * *)]$ waren 7 symmetrische (14\% der Gesamtzahl) und diese nicht vollkommen exakte, nur angenäherte, während $39(78 \%)$ mebr oder weniger ausgesprochene Asymmetrie zeigten, welche Asymmetrie wie aus den Tabellen ersichtlich ist -- nur in einer geringen Anzabl von Fällen $4(8 \%)$ auf Konvergenzasymmetrien zurückzuführen ist. Wenn wir daher unter den 46 Heterophorien 45 als alternierend, 1 als nicht alternierend bezeichnet haben, so gilt das nur dem Typus nach, dem Sinne der Ablenkung nach, nicht aber dem quantitativen Zahlenverhältnisse nach. Bei dieser Einteilung wurden übrigens nur die Breitenabweichungen berücksichtigt, nicht aber die Höhenabweichungen; sonst würde sich die Zahl zugunsten des nicht alternierenden Typus verschieben, weil

*) Wir können Bielschowsky ${ }^{23}$ ), S. 166 also nicht beistimmen, daß Konvergenzinsuffizienzen bei ganz Gesunden nur gelegentlich vorkommen, denn unser untersuchtes Studentenmaterial bestand aus ausgesuchten gesunden Individuen.

**) Diese Ansicht wurde sehon seit langem von A. Tschermak vertreten.

****) Dolmann ${ }^{27}$ ) fand mit einem eigenen Verfahren an 100 Emmetropen bei $66 \%$ eine größere, bei $7 \%$ eine kleinere Ablenkung, bei $17 \%$ eine symmetrische Heterophorje. Unausgeglichene Refraktionsfehler und mangelnde Kopffixation bezeichnet er selbst als Fehlerquellen. Banister ${ }^{28}$ ) hält die in $80 \%$ bestehende leichte Heterophorie für keine ernste Störung. 
die Höhenabweichungen beider Augen öfters gleichsinnig gefunden wurden, manchmal überhaupt nur ein Auge eine Höhenabweichung erkennen ließ. Solche Fälle sind also offenbar nicht so selten. Asymmetrien sind auch hier wieder vorherrschend. Unter den Rollungs. abweichungen waren alternierend 3 , einseitig 4 .

Es ist nun sehr wohl möglich, daß sich bei Untersuchung in größeren Entfernungen noch einige angenäherte Orthophorien herausfinden lassen würden (von den $50 \%$ mit suffizienter Konvergenz ist es bezüglich Seitenabweichungen nicht sehr wahrscheinlich), daß also unsere Zah! der Heterophorien etwas zu hoch gegriffen erscheint. Es sollen und können die gegebenen Zahlenangaben auch keine fixe Statistik darstellen, dazu ist die Zahl der Untersuchten viel zu gering, - abgesehen von einer Anzahl anderer Komplikationen, die später gelegentlich der Mitteilung der Eigenbeobachtungen des Verfassers berichtet werden. Unter anderem ist das wichtigste: Alle die Erscheinungen sind zeitlich nicht konstant, sondern sind unter Umständen sogar relativ großen Schwankungen unterworfen. Alle Zahlenangaben über Quantität und ertl. sogar Sinn der Heterophorien sind also zumindest sehr problematisch! Eines jedenfalls zeigen die bisherigen Untersuchungen: Vollkommene Symmetrie und korrektes Zusammenarbeiten des Doppelauges ist überaus selten nach Ausschaltung der Fusionsreize; die Fusion ist das überaus wichtige Korrektionsmittel, welches ein tadelloses Zusammenarbeiten des Doppelauges ermöglicht.

\section{b) Spezialreihe von Selbstbeobachtungen.}

Nun sei noch über eine Anzahl zumeist eigener Beebachtungsreihen in aufsteigenden Distanzen von 0,5 bis $6 \mathrm{~m}$ und umgekehrt in absteigenden Entfernungen berichtet. Verfasser selbst hat beim Fernsehen eine Esophorie (hier sollen der Einfachheit halber nur die Horizontalabweichungen berücksichtigt werden), die beim Nahesehen in eine Exophorie (Insuffizienz der Konvergenz) umschlägt. Derartige Fälle sind öfters beschrieben worden, unter anderen neuestens von Andrews ${ }^{35}$ ) bei Siebbeinerkrankung, von Banister ${ }^{43}$ ), bei Hysterie als relativ häufig von Bielschowsky ${ }^{23}$ ). Die Versuchsbedingungen während der einzelnen, auf- und absteigenden Reihen wurden möglichst konstant gehalten, das verdeckte Auge blieb immer genau $2^{\prime}$ unter VerschluB, die Versuchszeiten aber wurden möglichst verschieden gewählt, nach vollkommener Frische früh nach dem Aufstehen, am Tage mitten in der Beschäftigung (zumeist Naharbeiten, Lesen, Schreiben usw.) und schließlich nach anstrengender Arbeit am Abend. Die Untersuchungen dehnten sich über einen Zeitraum von etwa 2 Monaten aus. Dabei ergab sich die unseres Wissens bisher nirgends erwähnte interessante Tatsache, daß die Abweichungen des abgeblendeten Auges zeitlich eine - oft recht er- 
hebliche - Inkonstanz aufweisen. Ganz besonders wechselnd sind die Höhenabweichungen, die nicht nur ihrer Größe nach, sondern auch in ihrer Richtung stark schwankten; am häufigsten traten nichtalternierende Höhenabweichungen [Bielschowsky $\left.{ }^{11}\right)$ ] auf, und zwar im Sinne einer positiven Vertikaldivergenz (Hyperphorie) bei abgeblendetem rechten Auge und einer negativen Vertikaldivergenz (Hyperphorie) bei abgeblendetem linken Auge - Vertikalabweichungen, die dann innerhalb einer relativ kurzen Versuchsreihe des öfteren gleich blieben, aber anch schwankten. Manchmal waren die Höhenabweichungen von alternierendem Typus, und zwar sowohl positive als auch negative; andere Reihen ließen wiederum Vertikalabweichungen nur für das eine Auge im positiven oder negativen Sinne erkennen. Hie und da erwies sich der Sinn der Vertikaldivergenz abhängig von der Beobachtungsentfernung. Wenn nun schon der Richtung nach sich nichts Bestimmtes finden ließ, so gilt das um so mehr von den Größenwerten, dje überhaupt ausgesprochen variabel waren. Auf die Rollungsabweichungen wurde bei diesen Reihen weniger Gewicht gelegt. Die Horizontalabweichungen fanden sich durchweg im Sinne von Esophorie beim Fernesehen und Exophorie beim Nahesehen mit Ausnahme einer einzigen Untersuchungsreihe (am 9. X. 1921) unmittelbar früh nach ausreichendem guten Schlafe, wie ich sie aber ein zweites Mal nicht mehr beobachten konnte. Damals ergab sich beim Nahesehen es war eine aufsteigende Reihe - die typische Exophorie, die für das reehte abgedeckte Auge bei $2 \mathrm{~m}$ Beobachtungsdistanz in eine reine Orthophorie umschlug, dann aber bis zu $6 \mathrm{~m}$ wieder in wechselndem Größenausma Be zutage trat; das linke Auge ließ bis zu $2 \mathrm{~m}$ Beobachtungsdistanz gleichfalls Exophorie erkennen, dann bis zu $5 \mathrm{~m}$ gar keine Seitenabweichung, nur negative Vertikaldivergenz (Hyperphorie), von $5 \mathrm{~m}$ an äußerte sich aber wieder eine deutliche Exophorie. Die übrigen Versuchsreihen gestatten etwa folgendes Schema aufzustellen [Abb, 10\%)].

In Wirklichkeit liegen die Doppelbilder bei dieser Darstellungsweise so gut wie nie (höchstens ganz ausnahmsweise angenähert) auf einer Geraden, sondern vielmehr auf einer variablen. Kurve, die 'oft Zickzackcharakter bzw. Richtungswechsel aufweist. Varia bel ist auch der Winke! den die Doppelbilderkurve xait der Geraden der Fixationspunkte einschließt. Sofort auffallend ist die Asymmetrie der beiden. Figuren, bei mir liegen die Verhältnisse so, daß im allgemeinen der Umschlagspunkt (die Entfernung - ein distinkter Punkt - oder die Entfernungen

*) Die Figuren stellen die bei den verschiedenen Beobachtungsdistanzen gefundenen Doppelbilder dar, die so hintereinandergereiht sind, da $B$ die Fixationspunkte des jeweils fixierenden Auges a uf einer Geraden liegen. Die Ordinaten zeigen die Beobachtungsentfernungen, die Abszissen die Doppelbilderdistanzen. Maßstab mehr oder weniger willkürlich, die Abszissenwerte stark übertrieben. 
- eine bestimmte Strecke -, wo das abgedeckte Auge bei Freigabe keine Seitenabweichungerkennen läßt) für das rechte Auge in einer größeren Beobachtungsdistanz (etwa $2 \mathrm{~m}$ ) liegt als für das linke Auge (etwa $1 \mathrm{~m}$ ). Zum Vergleiche mit obigem Schema sei eine bestimmte Beobachtungsreike vom 31. X. 1921 herausgegriffen und nicht schematisiert dargestellt [Abb. 1l*)].

Bemerkenswert daran ist vor allem, daß das rechte Auge in Distanzen von 1-2m gar keine Seitenabweichungen zeigt.

Abgesehen davon, daß die Umschlagspunkte (-strecken) für beide Augen in verschiedenen Entfernungen liegen, ist aber auch von Wichtigkeit, daß die Umschlagspunkte (-strekken) jedes einzelnen Auges zeitlich in of trecht variablen Beobachtungsentfernungen anzutreffen sind, ein Beweis dafür, daß die Verhältnisse an den Augenmuskeln zeitlich erheblichen Veränderungen

*) Bei dieser Darstellung sind nicht nur die Seitenabweichungen, sondern auch die Höhenabweichungen der Doppelbilder berücksichtigt. Die Doppelbilderdistanzen sind im Vergleiche zur Kreuzesgröße im Verhältnisse von $2: 1$ eingezeichnet.

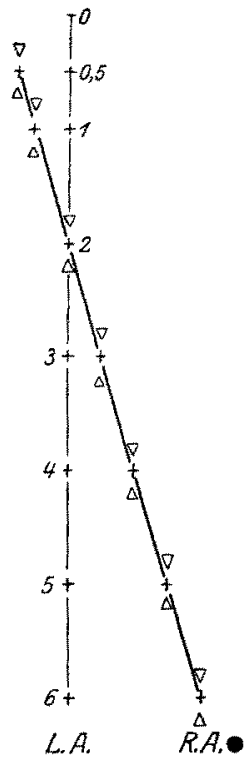

Linkes Auge fixierend, rechtes Auge abgedeckt. Abb. 10

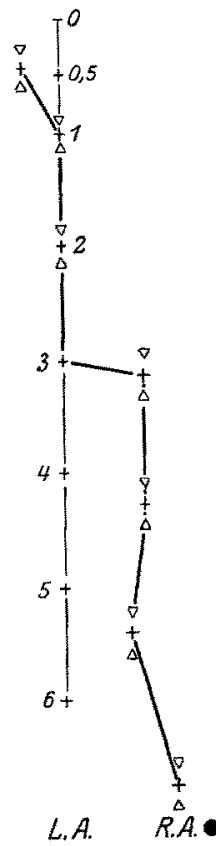

Linkes Ange fixierena, rechtes Auge abgedeckt. $A b b, 11$.

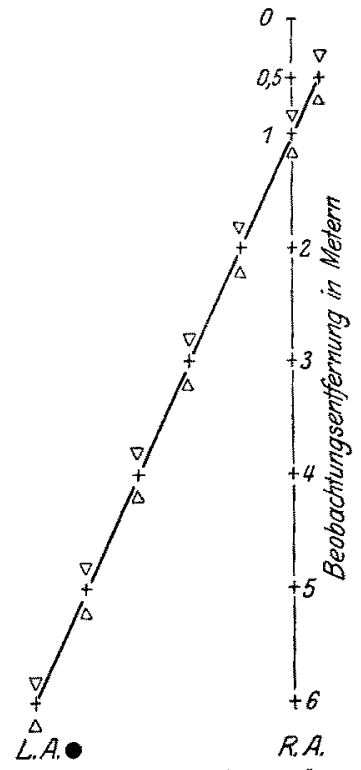

Rechtes Auge fixierend, linkes Ange abgedeckt.

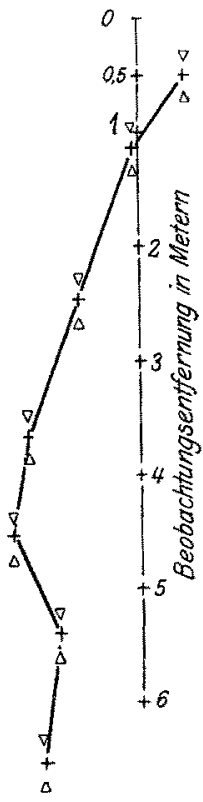

L.A. R.A.

Rechtes Auge fixierend, linkes Auge abgedeckt.

จ. Graefes Arehiv für Ophthalmologie. Bd. 108. 
unterworfen sind. Die folgende graphische Darstellung [Abb. 12*)] soll dieses Verhalten erläutern:

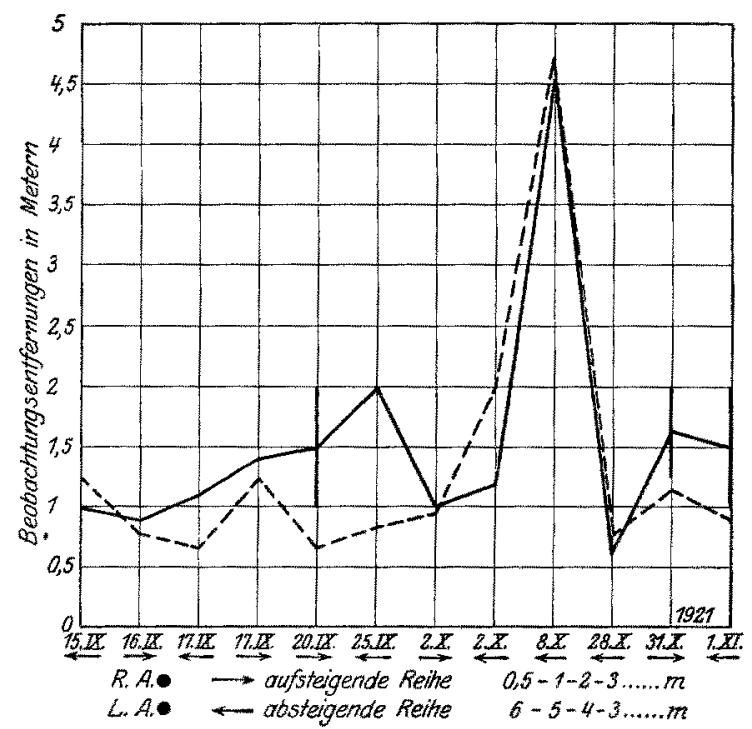

Abb. 12.

Am auffallendsten ist das weite Hinausrücken des Umschlagspunktes beider Augen bei der Beobachtungsreihe vom 8. X. 1921. Dieser Fall ist in gewissem Sinne analog zu dem kurz vorher beschriebenen Ausnahmsfall, bei welchem überhaupt kein Umschlag auftrat (derselbe ist in die Kurve nicht aufgenommen); diese Reihe wurde gleichfalls unmittelbar nach dem Aufstehen ausgeführt.

e) Grundlagen des Variierens der Abblendungsstellung.

Mehrere Faktoren sind es offenbar, welche für dieses wechselnde Verhalten der Stellung des abgeblendeten Auges in Betracht kommen: Das Zentralnervensystem [siehe u.a. Walter ${ }^{42}$ )] an sich als efferentes tonusvermittelndes und alteratives Zentrum, weiter alle jene Vorgänge, welche nachweislich reflektorisch den Tonus der Augenmuskeln beeinflussen können, so das Licht, das Labyrinth, die Halsmuskulatur und schließlich Eigenschaften des Muskels selbst.

In Hinsicht auf den Zustand des Zentralnervensystems war neben einer anderen Anzahl ron Beobachtungen besonders das Verhalten eines von mir untersuchten

*) Auf den Abszissen sind die Beobachtungstage registriert, auf den Ordinaten sind die Umschlagspunkte in den verschiedenen Beobachtungsdistanzen eingezeichnet. Sind Umsohlagsstrecken gegeben (nur beim rechten Auge), so verbindet die Kurve den Mittelpunkt derselben. 
Studenten bemerkenswert, den ich das erstemal im Februar 1921 eine Reihe ausführen ließ, als er sich im. Prüfungsstadium des $\mathrm{X}$. Rigorosums befand und einen sichtlich angestrengten, neuropathischen Eindruck machte; damals fand sich für das R.A. - bei $50 \mathrm{~cm}$ eine Exophorie von $11^{\circ} 45^{\prime}$, bei $100 \mathrm{~cm}$ von $6^{\circ} 24^{\prime}$, für das L. A. bei $50 \mathrm{~cm}$ eine Exophorie $\operatorname{von} 11^{\circ} 45^{\prime}$, bei $100 \mathrm{~cm}$ von $8^{\circ}$. Nach bestandenen Prüungen und besonders der langen dreimonatigen Erholungspause der Hauptferien, Anfang November neuerlich geprüft - subjektiv erschien er wesentlich ruhiger - zeigte er unter sonst gleichen Bedingungen nur mehr folgende Exophorie: R. A. - bei $50 \mathrm{~cm} 5^{\circ} 43^{\prime}$, bei $100 \mathrm{~cm} 4^{\circ}$, L. A. - bei $50 \mathrm{~cm} 6^{\circ} 5 \mathrm{I}^{\prime}$, bei $100 \mathrm{~cm} 5^{\circ} 9^{\prime}$. Schließlich spricht auch der veränderte Ausfall der Untersuchung nach ausreichendem Schlafe in demselben Sinne.

Bei einer andern Person, die gleichfalls 2 mal mit mehrmonatiger $Z$ wischenpause untersucht wurde, war das Verhalten ganz ähnlich dem eben geschilderten. Selbstredend hängt vom Zustande des Zentralnervensystems (Ermüdung usw.) auch das Konvergenz-Divergenzvermögen ab. Ein tonischer Einfluß der Belichtung dèr Netzhäute auf die Augenmuskeln ist heute so gut wie sichergestellt $\left.\left[O \mathrm{hm}^{44}\right)\right]$. Dafür sprechen, abgesehen von anderen Komplikationen die Befunde von F. W. Marlow ${ }^{33,34}$ ), der bei einer Anzahl von Patienten die vorhandene Exophorie durch mehrere (3) Wochen hindurch geübte Verdunkelung des einen Auges bis auf einen dann recht konstant bleibenden relativ hohen Wert steigern konnte; der Autor weist auf die Bedeutung des längerdauernden Ausschlusses des einen Auges bei der Prüfung auf Heterophorie nachdrücklichst hin. Hierher gehören anch die von Bielschowsky ${ }^{46}$ ) und Lohmann ${ }^{29}$ ) beobachteten eigenartigen Bewegungsphänomene; dieselben fanden, daß amblyopische *) und amaurotische Augen (phthisische Stümpfe) durch Verdeckung (Verdunkelung) des sehtüchtigen Auges ihre Stellung änderten. Diese Bewegungserscheinungen sind wohl kaum als Mitinnervationen neben der auftretenden Mydriasis [ Lohmann $\left.{ }^{29}\right)^{* *}$ )] zu erklären, sondern etwa so, daß die Freigabe des normalen Auges durch die Belichtung zu einer sog. "Weckungsstellung“ (nach A. Tschermak) des anderen Auges führt.

SchlieBlich wäre hier noch der von einer Anzahl von Autoren beschriebene Dunkelnystagmus [zusammengestellt bei Bartels $\left.{ }^{32}\right)$ ] ins Treffen zu führen.

Uber den tonischen Einfluß des Labyrinthes und der Halsmuskulatur auf die Stellung der Augen ist in den letzten 10 Jahren eine große Anzahl von ausgezeichneten Untersuchungen an Tieren (besonders Kaninchen

*) Bei amblyopischen, wenigstens zum Teile noch über qualitatives Sehen verfügenden Augen wäre übrigens daran zu denken, ob nicht doch noch die Fusionsreize einen Einfluß haben könnten!

**) In einer späteren Arbeit [Lohmann $\left.\left.{ }^{39}\right)\right]$ ist derselbe übrigens selbst gelegentlich der Mitteilung von fünf ähnlichen Fällen von seiner ursprünglichen Auffassung abgekommen und schreibt diese Bewegungsphänomene dem Einflusse der Belichtung zu. 
und Katzen) im Pharmakologischen Institut zu Utrecht von R. Magnus und seiner Schule ausgeführt worden; erwähnt seien nur die Arbeiten von A. de Kleijn und R. Magnus ${ }^{36,37}$ ) und von A.de Kleijn ${ }^{38}$ ), die einigermaßen zusammenfassende Darstellungen bieten. Auf meine Untersuchungen am Menschen [M. H. Fischer $\left.\left.{ }^{21}\right)\right]$, deren Resultate trotz der gänzlich verschiedenen Methodik sehr interessante Parallelen zu den eben genannten bilden, wurde schon früher hingewiesen. Schon dort wurde bei der Besprechung der "Ruhelage" nachdrücklich darauf aufmerksam gemacht, daß die Tonusverteilung an den Augenmuskeln keine konstant gleichbleibende sein kann. Geringe Kopfstellungsänderungen, desgleichen Lageänderungen des Kopfes zum Körper bei derselben absoluten Lage des Kopfes im Raume sind imstande, Änderungen der Spannungsverteilung an den Augenmuskeln zu bewirken. Es ist auch keineswegs zu erwarten, daß etwa unter sonst gleichen Bedingungen bei einem Individuum zeitlich die Tonusverteilung die gleiche bliebe. Schließlich könnte auch die evtl. myogene Komponente des Muskeltonus Schwankungen unterworfen sein. Noch ein Faktor darf nicht unerwähnt bleiben, daß vielfach Muskeln nach alterativer Innervation einen lange nachdauernden Kontraktionsrückstand oder allgemein gesagt eine anhaltende Verkürzungslage aufweisen, ähnlich wie es Hofmann und Bielschowsky $y^{14}$ ) bezüglich der Rollungen nachgewiesen haben. Wenn nun jemand sich tagsüber fast durchweg mit Naharbeit (Konvergenz) beschäftigt, so ist es begreiflich, daß seine Augen eine andere relative Konvergenzlage infolge solcher Kontraktionsrückstände oder Dauerverkürzungen beibehalten können, wenn auch das eine Auge eine Abdeckung erfährt*); während des Schlafes kann dann der Ausgleich wieder stattfinden. Für das würden unter anderem bei meinen Beobachtungen die 2 Reihen sprechen, wo einmal unmittelbar nach dem Aufstehen überhaupt keine Esophorie auftrat, das andere Mal der Umschlagspunkt erst sehr weit draußen lag. Vor diesen. Beobachtungen wurde Naharbeit absichtlich vermieden. Hierher wären auch wiederum in gewissem Sinne die Befunde F. W. Marlows ${ }^{33}$, 34) bei langdauernder Abdeckung eines Auges zu rechnen.

Aus allen diesen Erörterungen ist ersichtlich, daß die Abblendungsstelllung der Augen keine konstante sein kann, sie muß zum. Teile recht beträchtlichen Schwankungen unterworfen sein. Es muß die zeitliche

*) Eine ähnliche Anschauung haben Banister und Lemeres [bei Banister $\left.\left.{ }^{43}\right)\right]$ ausgesprochen; sie glauben, da 8 bei Fällen von gleichzeitigem Vorkommen von Esophorie mit Insuffizienz der Konvergenz durch die dauernde Konvergenzanspannung in den Interni ein Dawerspasmus entsteht, der eine Esophorie vortäuscht. $K a z^{45}$ ) fand bei Schulkindern nicht selten durch fehlerhafte Kopfhaltung (übermäBig nahes Heranrücken an die Bücher) Esophorie auftreten, die später eventuell in Exophorie umschlug. 
Konstanz derselben als ein relativ seltener Ausnahmsfall bezeichnet werden. Darum haben alle Zahlenangaben überQuantität, aber auch Qualität derselben nur $G$ iultigkeit für den Zeitpunkt der Untersuchung und die dabei gegebenen Bedingungen. Selbst unter sonst gleichen physikalischen. Verhältnissen (AuBenfaktoren, Reizfaktoren) ist keine Gleichheit der Abblendungsstellung garantiert, es müssen eben hier wie bei allen biologischen Prozessen die variablen physiologischen Faktoren des Individuums selbst berücksichtigt werden. Als wichtigste Konsequenz daraus ergibt sich, daB die Resultate selbst wiederholter Heterophorieuntersuchungen nicht die ausschließliche Grundlage für eine rein anatomische Korrektur einer bestehenden Heterophorie bilden können und dürfen*); es ist kein Wunder, wenn solche Müskelverlagerungen so häufig - wie ja vielerseits die Erfahrung gemacht wurde - Versager aufweisen. Selbstredend wird es auch Fälle geben, wo die Schwankungen nicht so ausgesprochen sind, Muskeloperationen den gewünschten Erfolg bringen werden. Was für den normalen Binokularsehenden recht ist, ist für den Sohielenden billig, auch da gelten ähnliche Verhältnisse. A. Tschermak**), welcher als Theoretiker die praktischen Okulisten immer wieder auf diese Momente verweist, vergleicht die Tenotomie oder die Vorlagerung eines Augenmuskels nur mit der anatomischen Ermöglichung einer anschlieBenden funktionellen Korrektur im Sinne orthopädischer UUbungstherapie. Auch Bielschowsky ${ }^{40}$ ) weist mit Recht darauf hin, daß volle Erfolge von Schieloperationen, die dem Schielenden zu dauerndem, mühelosem Binokularsehen verhelfen, nur in einem relativ kleinen Prozentsatz zu verzeichnen sind. Häufig verhindert die Unbeständigkeit des Schielwinkels in seiner Abhängigkeit von physischen und psychischen Faktoren einen Dauererfolg. Auf die Frage der Brillen- bzw. Prismenkorrektur soll hier nicht näher eingegangen werden.

Eine um so größere Aufmerksamkeit muß dem reflektorisch wirksamen Ausgleichsmechanismus zugewendet werden, der unter normalen Umständen alle Ungleichmäßigkeiten in Bau und physiologischer Funktion der Augenmuskeln der beiden Hälften unseres Sehorgans verdeckt, uns dieselben nicht zum Bewußtsein kommen läßt und den Eindruck hervorruft, als ob von vornherein ein tadelloses Zusammenarbeiten, eine exakte Symmetrie gegeben wäre: dem Fusionsmechanismus. Es liegt nahe, den physiologischen Fusionsvorgang in Vergleich zu setzen mit dem Kontraste, der als physiologisches Korrektionsmittel die $\mathrm{Ab}$ bildungsfehler bzw. die unvermeidliche Astigmatik des dioptrischen

*) O'Connor ${ }^{41}$ ) steht auf dem Standpunkte, da B Operationen nicht gemacht werden sollen, um Heterophorien, zu beseitigen, sondern um die Beschwerden zu vermindern, was sicher in einer größeren Anzahl von Fällen erreichbar ist.

**) Unter anderem: Besprechung des Buches von $H$. Parinaud, „Le strabisme et son traitment". Zentralbl. f. prakt. Augenheilk. 1900, S. 209-213. 
Apparates (gegeben durch die Inhomogenität, den Zentrierungsmangel und die Irregularität der Krümmung der brechenden Medien, sowie durch die chromatische, sphärische und diffraktive Aberration) verdeckt, das Auge als exakt arbeitendes optisches System erscheinen läßt, indem er eine Stigmatik (Schärfe) der Enderregungsvorgänge und damit der Empfindungseffekte vermittelt. Die auf der fixen, elementaren sensorischen Korrespondenz ruhende Fusion bewirkt - wenn man so sagen darf - erst die Stigmatik in der Einstellung des motorischen Bewegungsapparates. Die Fusion stellt offenbar einen Reflexvorgang besonderer Art dar, der zum binokularen Einfachsehen führt. E. Hering ${ }^{2}$ ) S. 17, $\left.38,{ }^{4}\right)$ S. 320, schreibt: „Die Augenbewegungen dürfen, wie sehr sie auch in zahllosen Fällen den Einđruck des Unwillkürlichen machen, nicht mit den reinen Reflexbewegungen zusammengeworfen werden, aber sie sind mit letzteren durchaus in eine gewisse Analogie zu bringen. Erst ein Ereignis des Bewußtseins, die Aufmerksamkeit macht das Netzhautbild zu einem Reflexreize." So auch hier; der Fusionsausgleichsmechanismus ist ein unwillkürlich arbeitender Reflexapparat, jedoch gebunden an das Intaktsein spezifischer, physiologischer und psychiseher Einrichtungen, die zum binokularen Einfachsehen drängen; es handelt sich um einen komplexen Vorgang. Sehr interessante Schädigungen des Fusionsapparates bei Hysterie mit ihren häufigen psychischen Störungen hat Bielschowsky $y^{23}$ ) beschrieben. Daß auch hier die Fusion vermittelnden Korrektivbewegungen den von $E$. Hering aufgestellten Gesetzen der assozijierten bilateralen. Augen bewegungen unterworfen sind, hat derselbe dort ausgeführt. Dennoch darf auch hier die Möglichkeit einseitiger Korrektionsbewegungen nicht von der Hand gewiesen werden. Existiert doch bei Hunden and Katzen, wio Hitzigis) und $\left.\$ i l e x^{31}\right)$ gefunden haben, 4. Tschermak $\left.{ }^{9}\right)^{*}$ ) ausdrücklich betont und noch nicht veröffentlichte Arbeiten im hiesigen. Institute bestätigt haben, im oberen Teile des Gyrus coronalis ein streng einseitig, und zwar kontralateral wirksames Rindenzentrum, welches jenem des Augenfacialis angegliedert erscheint. Beim Menschen ist eine solche Rindenpartie bisher allerdings nicht nachgewiesen, doch ist ihre Existenz gewiß nicht unwahrscheinlich; zum mindesten muß mit deren Möglichkeit gerechnet werden. Über die funktionelle Bedeutung dieses Rindenzentrums ist allerdings noch nichts festgestellt, wenn anch eine kontralaterale Korrektur gewisser Asymme-

*) A. Tscherma $k^{9}$ ) unterscheidet neben diesem einseitigen okulomotorisohen Zentrum drei Rindenzentren für assoziierte Augenbewegungen, welche auch für den Menschen sichergestellt sind, und zwar das frontale oder präzentrale Spähzentrum ohne direkte optische oder alkustische Wahrnehmungsgrundlage, dann das optische occipitale Blickzentrum in der Sehgphäre bzw. in der Calcarinarinde für Bliokbewegungen auf optische Findrucke hin (Verfolgungsbewegungen des Blickes), endlich das akustische insulotemporale Blickzentrum in der Hörsphäre "ozw. in der Querwindung der Insel für Bliekbewegungen auf akustische Eindrücke hin. 
trien des okulomotorischen Apparates plausibel erscheint. Jedenfalls ist es geratener, mit dieser naheliegenden Möglichkeit zu rechnen, als zu gewagten Hilfshypothesen zu greifen, um eine ausnahmslose und unbedingte Bilateralität der Augenmuskelinnervation aufrechtzuerhalten.

Zweifellos können die durch die Fusion ausgelösten Korrektionsbewegungen durch Willensakte eine Unterstützung erfahren, wenn es sich um den Ausgleich von Lateralabweichungen handelt, der durch Konvergenzmehrung bzw. -minderung beschleunigt werden kann. Umgekehrt gibt es auch eine große Anzahl von allerdings meist besondersgearteten Individuen*), die willkürlich entgegen dem Fusionszwange das Auftreten der Ausgleichsbewegungen verhindern können, allerdings nicht unter jeder Bedingung. Bei der weitaus überwiegenden Mehrzahl (so wenigstens war es bei unserem Materiale) sind die Fusionsbewegungen dem Willen aber vollständig entzogen, wobei allerdings nicht in Abrede gestellt werden kann, daß sie durch Schulung und Ubung doch noch willkürlich beeinflußt werden könnten. Dann sind freilich nicht mehr die ursprünglichen natürlichen Verhältnisse gegeben, sondern mehr oder weniger künstlich modifizierte. Unsere Versuchspersonen verhielten sich (mit 2 Ausnahmen [4\%]!) so, daß die sofort eintretende Korrektionsbewegung absolut nicht gehemmt werden konnte, oder daß überhaupt jede Ausgleichsbewegung ausblieb und auch willkürlich nicht hervorgerufen werden konnte; zu letzterer Gruppe gehörten in erster Linie Individuen mit Heterophorien von über $3^{\circ}$ bei $100 \mathrm{~cm}$ Beobachtungsdistanz. Wohl aber rückten die Doppelbilder bei jedem willkürlichen Lidschluß in diesen Fällen schrittweise näher und näher, bis sie überhaupt verschwanden. Aber auch solchen Personen, die gelernt haben (ich selbst gehöre hierher), die Korrektionsbewegungen willkürlich zu hemmen (die also die Doppelbilderdistanz festhalten können), wird unter bestimmten Verhältnissen jeder Willenseinfluß auf dieselben entzogen - wie folgender Fall lehrt.

Wenn ich des öfteren abends am Moldauufer spazierenging, machte ich mir das Vergnügen, die gleichmäßigen Reihen der Bogenlampen, die über die Brücken ziehen, mit starker falscher Konvergenz (Konvergenz auf einen näheren Punkt als die Lampen) anzusehen; man bekommt dann den äußerst vergnüglichen Eindruck, als schwebe eine Reihe von kleinen Bogenlämpehen in ganz unmittelbarer Nähe vor den Augen. Beginnt man mit dem Experiment, so kann man, solange die gleichseitigen Doppelbilder noch relativ weit voneinander abstehen, dieselben ziemlich lange halten; hat aber die Doppelbilderdistanz einmal einen bestimmten Wert unterschritten (und zwar sowohl in bezug auf die Ausgangsstellung als auch auf eine neue Konvergenzstellung), so schnappen sie geradezu ineinander; man

*) Hier handelt es sich zumeist um Leute, die sich häufig unokular beschäftigen oder ganz speziellen optischen Versuchen nachgehen oder -gingen, infolgedessen ihrem okulomotorischen Apparate eine ganz besondere Aufmerksamkeit zuwenden. 
spürt einen unwiderstehlichen Zug an den Angenmuskeln, das Einschnappen 1 äBt sich durch nichts mehr hemmen. Ganz genau dasselbe konnte ioh bei Betrachtung der von R. Er. Kahn ${ }^{4 i}$ ) beschriebenen Tapetenbilder beobachten; bei seitlicher Kopfwendung spuirt man wiederum sehr deutlich den Zug an den Augenmuskeln. Kahn selbst, der bei unserer Versuchsanwendung auch die Doppelbilder willkürlich festhaIten konnte, schreibt: „Der Zwang zum Einfachsehen bei nicht ganz kongruenter Lage der einzelnen Details des Musters ist ein sehr starker." Fon Pref. R. H. $K a h n$ wurde ich erst auf diese Erscheinung bei den Tapetenbildern a ufmerksam gemacht.

Wie schon erwähnt warde, scheiden wir die Fusionsanspruchsfähig. keit von der Fusionsbreite.

Die Fusionsbreite ist durch den größten von den Doppelbildern und dem Drehpunkte des Zyklopenauges eingeschlossenen Winkel bestimmt, bei welchem eben gerade noch der Ausgleichsmechanismus einsetzen kann; ist die Fusionsbreite überschritten, so fehlt jeder Ausgleich, ja es kann unter Umständen sogar die Doppelbilderdistanz zunehmen. Auch die Fusionsbreite ist offenbar abhängig von den Versuchsbedingungen, wie schon in der Anmerkung S. 268 ausgeführt wurde; es spielen hier wohl die Deutlichkeit, Beleuchtungsstärke, die Gestalt der Doppelbilder eine große Rolle, verschiedene Beleuchtung der beiden. Augen, Refraktionsunterschiede, verschiedene Grade der Sehtüchtigkeit usw. Auch die Fusionsbreite ist sicher (infolge Ermüdung usw.) eine variable Größe, was allerdings erst weitere Untersuchungen zeigen müßten.

Die Fusionsanspruchstähigkeit ist ein Maß für die Schnelligkeit des Einsetzens des Ausgleichsmechanismus; sie ist individuell sehr verschieden ausgeprägt. Zwischen Fusionsbreite und -anspruchsfähigkeit bestehen gewöhnlich - doch nicht zwangsläufig - derartige Beziehungen, daß Individuen mit relativ großer Fusionsbreite eine geringe Anspruchs. fähigkeit besitzen (die Korrelktivbewegung setzt ganz allmählich ein und führt allmählich zum Binokular-Einfachsehen), hingegen Individuen von relativ geringer Fusionsbreite eine ausgezeichnete Fusionsanspruchsfähigkeit a ufweisen, die einen sehr raschen Ausgleich garantiert. Doch gibt es, wie wir beobachten konnten, Ausnahmefälle.

Wäre es in Hinsicht auf die enorme Bedeutung der Fusionsvorgänge möglich, sie therapeutisch zu beeinflussen, könnte man diesen so überaus wichtigen korrektiven Faktor gewissermaßen, fördern, abstufen, so würde man sicher oft überraschende Erfolge sehen. Wenn auch die Auffassung Worths ${ }^{49}$ ) nicht in dem MaBe zutrifft, daB Mangel (Bchwëche) des Fusionsvermögens einen weitaus überwiegenden Hauptfaktor in der Genese des Schielens bildet, demgegenüber alle anderen Faktoren als untergeordnet zurücktreten, so gebührt ihm doch das Verdienst, die enorme Bedeutung dieses Korrektivmechanismus auf das nachdrücklichste betont zu haben. 


\section{4. Übersicht der Ergebnisse.}

1. Der Begriff Primärstellung ist für die kinematisch ausgezeichnete, durch das Listingsche Gesetz charakterisierte Augenstellung zu reservieren. Dieselbe entspricht nicht der absoluten oder einer relativen Ruhelage des Auges, sondern einer davon einigermaßen abweichenden Zwangslage.

2. Als sog. Ruhelage des Einzelauges sei eine Stellung entsprechend dem subjektiven Empfindungseindrucke von Geradevorne und Gleichhoch bezeichnet. Dieselbe scheint nicht weitgehend verschieden zu sein von der absoluten oder anatomischen Ruhelage, immerhin aber einer tonischen Gleichgewichtslage von minimaler Tonusverteilung zu entsprechen.

3. Die Abblendungsstellung des Einzelauges entspricht einer Zwangslage von höherem, ungleichmäßig verteiltem Spannungstonus der Augenmuskeln. Thre Größenordnung; ja ihr Abweichungssinn ist nicht konstant, sondern zeigt je nach Zustandslage des Individuums charakteristische Verschiedenheiten. Für das einzelne Individuum ergaben sich eventuell in Mehrzahl Umschlagspunkte je nach dem Beobachtungsabstand, die jedoch selbst variieren.

4. Die Abweichung der Abblendungsstellung von der idealen Einstellung - die sog. Heterophorie - wurde mittels einer besonderen einwandfreien Methode (Binokularsichtbarkeit der Fixiermarke mit unokularer Kennzeichnung des einen temporär abblendbaren Auges) messend verfolgt. Für die Bezeichnung dieser Abweichungen nach Lateral-, Vertikal- und Rollungskomponenten wird eine präzise Nomenklatur vorgeschlagen.

Unter 50 Studenten wurden bei den gewählten Beobachtungsbedingungen bei Nahesehen auf $1 \mathrm{~m}$ nur 4 fast vollkommene Orthophorien (bei Emmetropen), 46 Heterophorien u. zw. 25 unter den Emmetropen, 21 unter den durchweg heterophoren Ametropen gefunden. Die Heterophorien waren ganz vorwiegend, aber doch nicht durchweg und vollkommen alternierend und in erheblicher Mehrzahl asymmetrisch.

5. Als Grundlage für das zeitliche Variieren der Abblendungsstellung bzw. der Heterophorie ergaben sich Einflüsse des Zentralnervensystems, bzw. Schwankungen des neurogenen, evtl. auch des myogenen Tonus der Augenmuskeln, speziell ein reflektorischer Einfluß der Belichtung (Weckungsstellung), wie auch des Doppelbildereindruckes (adaptative Abwendungssteigerung der Heterophorie). Auch an einer reflektorischen Einflußnahme von Labyrinth und Kopf-Halsmuskulatur auf den Augenmuskeltonus bzw. die Heterophorie ist nicht zu zweifeln.

6. An Fusion ist Fusionsbreite und Fusionsanspruchsfähigkeit streng zu unterseheiden. Die letztere ist in der Regel bei geringer Fusionsbreite 
ausgesprochener. Die Fusion stellt -- analog der Bedeutung des Simultankontrastes für die Stigmatik der Anschauungsbilder - ein Korrektionsmittel dar für die Asymmetrien im okulomotorischen Apparate beider Augen und führt zur Stigmatilk in der motorischen Einstellung beider Augen gemäß ihrer fixen, funktionellen sensorischen Verknüpfung.

7. An Korrektivbewegungen kommen in erster Linie bilateral-assoziierte Impulse bzw. Bewegungseffekte in Betracht. Daneben ist jedoch die Möglichkeit einer kontralateral einseitigen Innervation, speziell Tonusbeeinflussung auch für den Menschen nicht auBer acht zu lassen, worauf die neuerdings bestätigte Auffindung eines kontralateral-einseitigen okulomotorischen GroBhirnrindenzentrums neben den drei Corticalzentren für assoziierte Augenbewegungen (frontales oder präzentrales Spähzentrum, optisches occipitales und alzustisches insulotemporales Blickzentrum) hinweist.

Meinem verehrten Lehrer, Herm Prof. A. Tschermak sei an dieser Stelle für seine gütige Förderung und seine Anregungen neuerlich mein herzlichster Dank ausgesprochen.

\section{Literaturverzeichnis.}

1) Hering, E., Raumsinn in Hermanns Handbuch d. Physiol. Bd. III. T. 1. 2) Hering, E., Die Lehre vom binokularen Sehen. Engelmann. Leipzig 1868. ) Hering, E., Über Muskelgeräusche des Auges. Sitzungsber. d. Wien. Akad. d. Wiss. 79, 3. Abt., 137-154. 1879. - 4) Hering, E., Zur Lehre vom Ortasinne der Netzhaut. Engelmann. Leipzig 1861. - 5) Tschermak, A., Der exakte Subjektivismus in der neueren Sinnesphysiologie. Pflügers Arch. f. d. ges. Physiol. 188, 1-20, auch separat, Springer, Berlin 1921. - 5) Tschermak, A., Übex die absolute Lokalisation bei Schielenden. v. Graefes Arch. f. Ophthalmol. 5.5, I, 1-45. 1902. 7) Tschermak, A., Über einen Apparat (Justierblock) zur objektiven Bestimmung der Pupillardistanz und zur Festsetzung der Stellung der Gesichtslinien. Pflügers Arch. f. d. ges. Physiol. 188, 21-24. 1921, - ${ }^{8}$ ) Tschermak, A., Utber physiologische und pathologische Anpassung des Auges. (Ein Vortrag.) Veit \& Comp. Leipzig 1900.$\left.{ }^{9}\right)$ Tschermak, A., Physiologie des Gehirnes in Nagels Handbuch der Physiol. Bd. IV. 1. Hälte, S. 1-206. Vieweg. Braunsohweig 1905. - 10a) Graefe, Alfred, Motilitätsstörungen. Graefe-Saemisch, Handbuch der ges. Augenheilk. Bd. VI, Kap. 9, 1880. - ${ }^{100}$ ) Graefe, Alfred, Motilitätsstörungen. Graefe-Saemisch, Handbuch der ges. Augenheilk. Bd. VIII. 2. Aull., T. 2, Kap. 11. 1898, - ${ }^{11}$ ) Bielschowshy, A., Utber die Genese einseitiger Vertikalbewegungen des Auges. Zeitschr. f. Augenheilk. 12, 545-557. 1904. - 12) Bielschoushly, A, und A. Ludwig, Das Wesen und die Bedeutung latenter Gleichgewichtsstörungen der Augen, insbesondere der Vertikalbewegungen. v. Graefes Arch. f. Ophthalmol. 6\%, 400-463. 1906. 13) Bielschowosky, A., Über latente Gleichgewichtsstörungen der Augen. Beriohte uber die 32. Versammlung d. ophthalmol. Ges., Heidelberg 1906, S. 25-33. 14) Hofmann, F. B. und A. Bielschotushy, Uber die der Willkir entzogenen Fusionsbewegungen der Augen. Pflügers Arch. f. d. ges. Physiol. 80, 1-40. 1900. - $\left.{ }^{15}\right)$ Fischer, O., Medizinische Physik. Hirzel. Leipzig 1913. S. 218-257, - ${ }^{16}$ ) Reddingizis A., Das sensumotorische Sehwerkzeug. Engelmann. Leipzig 1898. - 7) Graefe, Alfred, Kli- 
nische Analyse der Motilitätsstörungen des Auges. Berlin 1858. - ${ }^{18}$ ) Hitzig, E. Untersuchungen über das Gehirn. Hirschwald. Berlin 1874; Ges. Abhandlungen. Ebenda 1304. - 19) Hansen, Grut, Die Schieltheorien. Arch. f. Angenheilk. 29, 69. 1894. - 20) Hofmann, F. B., Einige Fragen der Augenmuskelinnervation. Ergebn. d. Physiol. 5, 599-621. 1906. - 21) Fischer, M. H., Messende Untersuchungen tiber das scheinbare Gleichhoch, Geradevorn und Stirngleich. Pflügers Arch. f. d. ges. Physiol. 188, 161-240. 1921. - 22) Schlodtmann, W. (unter A. Tschermak), Studien über a nomale Sehrichtungsgemeinschaft bei Schielenden. v. Graefes Arch. f. Ophthalmol. 51, 256-294.1900, ${ }^{23}$ ) Bielschousky, A., Die hysterischen und funktionellen Störungen der Augenbewegringen. Zentralbl. 1920. - ${ }^{24}$ ) Kleijn, A. de und R. Magnus, Über die Unabhängigkeit der Labyrinthreflexe vom Kleinhim und über die Lage der Zentren für die Labyrinthreflexe im Hirnstamm. Pflügers Arch. f. d. ges. Physiol. 178, 124_178. 1920. 둘) Maddox, E. E. und W. Asher, Die Motilitätsstörungen des Auges. Deichert. Leipzig 1902. 26) Baldino, Salvatore, Un nuovo appa rechio per la determinazione della eteroforia. Arch. di ottalmol. 27, 57-68. 1920. - $\left.{ }^{27}\right)$ Dolman, Perciral, The relation of the sighting eye to the measurement of heterophoria. A preliminary report. Americ. Journ. of ophthalmol. 31, 258-261. 1920. - ${ }^{29}$ ) Banister, J. M., Hints in relation to the dynamics of the extrinsic ocular muscles with suggestions as to treatment of states of muscular imbalance. Americ. Journ, of ophthalmol. 3, 878-883. 1920. - ${ }^{29}$ ) Lohmann, W., Über ein beobachtenswertes Bewegungsphänomen des Auges. Klin. Monatsbl. f. Augenheilk. 48, 556--559. 1910. - ${ }^{30}$ ) Graefe, Albrecht v., Symptomenlehre der Augenmuskellähmungen. Berlin 1867. - ${ }^{\text {11) }}$ Bois-Reymond, R. du und $P$. Silex, Über corticale Reizung der Angenmuskeln. Arch. f. Physiol. 1899, S. $\left.174-184 .{ }^{32}\right)$ Bartels, M., Aufga ben der vergleichenden Physiologie der Augenbewegungen. v. Graefes Arch. f. Ophthalmol. 101, 299-332. 1920. - ${ }^{33}$ ) Marlow; $F$.W., Prolonged monocular occlusion as a test for the musclebalance. Americ. Journ. of ophthalmol. 4, 238-250, 1921. - 34) Marlow, F.W., Transaction of the Americ. ophthalmol. soc., 56. ann. meet. 18, 275 -290. 1920. - ${ }^{35}$ ) Andrews, A. H., Heterophoria from ethmoid. disease. Transact. of the 25. ann. meet. of the Americ. acad. of ophthalmol. a. oto-laryngol. 1920, p. 11-18. - $\left.{ }^{36}\right)$ Kleijn, A. de und R. Magnus, Über die Funktion der Otolithen. I. Mitteilung. Otolithenstand bei den tonischen Labyrinthreflexen. Pflügers Arch. f. d. ges. Physiol. 186, 6-38. 1921. - ${ }^{37}$ ) Kleijn, A. de and R. Magnus, Über die Funktion der Otolithen. II. Mitteilung. Pflügers Arch. f. d. ges. Physiol. 186, 61-81. 1921. - ${ }^{38}$ ) Kleign, A. de, Tonische Labyrinthund Halsreflexe auf die Augen. Pflügers Arch. f. d. ges. Physiol. 186, 82-97. 1921. - ${ }^{30}$ ) Lohmann, $W$., UUber die mit der Verdunkelung bzw. Belichtung des Auges (mit dem Pupillenreflex?) verknüpften abnormen Bewegungen des Augapfels. Arch. f. Augenheilk. 76, 15-26. 1914. - ${ }^{40}$ ) Bielschowsky, A., Ungewöhnliche Ursachen von MiBerfolgen in der Therapie des Schielens. Arch. f. Augenheilk. 69, 1-29. 1911. - ${ }^{41}$ ) $O^{\prime}$ Connor, $R$., The possibilities of muscle operations. Americ. Journ. of ophthalmol. 3, 116-118. 1920. - ${ }^{{ }^{12}}$ ) Walter, Will, Heterophoria and heterotropia. Americ. Journ. of ophthalmol. 3, 201-205. 1920. - ${ }^{43}$ ) Banister, $J . M$., Practical considerations in connection with insufficiency of convergence of the visual axes. Americ. Journ. of ophthalmol. 3, 269-275. 1920. ${ }^{44}$ ) Ohm, $J$., Über die Beziehungen zwischen Sehen und Augenbewegungen. Zeitschr. f. Augenheilk. 43, 249 -263. 1920. - $\left.{ }^{45}\right) \mathrm{Ka}$, $R$., Heterophorie infolge fehlerhafter Haltung bei Schulkindern. Wochenschr. $\mathrm{f}$. Therap. u. Hyg. d. Auges 1\%, 133-134. 1914. - 46) Bielschousky, A., Über einseitige Augenbewegungen. Fortschr. d. Med. 1909, Nr. 24-25. - ${ }^{47}$ ) Kahn, R. H., Über Tapetenbilder. Arch. f. Anat. u. Physiol., Physiol. Abt., 1907, S. 56-66. - ${ }^{48}$ ) Kahn, R. H., Beiträge zur Lehre vom Muskeltonus: 1. Über den Zustand der Muskeln der 
284 M. H. Fischer: Beiträge und kritische Studien zur Heterophoriefrage usw.

vorderen Extremitäten des Frosches während der Umklammerung. Pflügers Arch. f. d. ges. Physiol. 17\%, 294-303. 1919. -- ${ }^{49}$ ) Worth, Claud, Das Schielen. Deutsche Ausgabe von E. H. Oppenheimer. Springer. Berlin 1905. ${ }^{30)}$ Fick, Adolf. Ztschr. f. rat. Medizin (3) IV. S. 101. 1853. - - - Ruete, Th., Ein neues Ophthalmotrop, Leipzig 185̃7. - å) Volkmann, A. W., Ber. d. sächs. Ges. d. Wiss. 6. II. 1869, S. 28. - ${ }^{53}$ ) Oppenheimer, H., Lehrbuch der Nervenkrankheiten, Bd. 2, S. 1032, Karger. Berlin 1905. - as) Bielschowsky, A., Uber die relative Ruhelage der Augen. Ber. über d. 39. Versammlung d. ophthaimol. Ges., Heidelberg 1913,67-78. - $\left.{ }^{-5}\right)$ Hoffmann, Paut, Uber die Aktionsströme der Augenmuskeln bei Ruhe des Tieres und beim Nystagmus. Arch. f. Anat. u. Physiol. Physiol. Abt., 1913, S. 22-34. 